\title{
Éducation arithmétique sous forme d' apprentissage. La géomancie dans les Monts Mandingues
}

Jan Jansen

\section{OpenEdition}

\section{Journals}

Édition électronique

URL : https://journals.openedition.org/etudesafricaines/16581

DOI : 10.4000/etudesafricaines. 16581

ISSN : $1777-5353$

Éditeur

Éditions de l'EHESS

\section{Édition imprimée}

Date de publication : 30 mars 2011

Pagination : $9-49$

ISBN : 978-2-7132-2297-9

ISSN : 0008-0055

\section{Référence électronique}

Jan Jansen, «Éducation arithmétique sous forme d' apprentissage. La géomancie dans les Monts Mandingues ", Cahiers d'études africaines [En ligne], 201 | 2011, mis en ligne le 05 mai 2013, consulté le 21 avril 2022. URL : http://journals.openedition.org/etudesafricaines/16581 ; DOI : https://doi.org/ 10.4000/etudesafricaines.16581

Ce document a été généré automatiquement le 21 avril 2022

(c) Cahiers d'Études africaines 


\section{Éducation arithmétique sous forme d' apprentissage. La géomancie dans les Monts Mandingues}

Jan Jansen

1 Cet $\operatorname{article}^{1}$ a sa justification, pour deux raisons. Tout $d^{\prime}$ abord, parce que $c^{\prime}$ est la première fois que ce système de géomancie est ainsi décrit, et ensuite parce qu' il s' agit d' une contribution théorique aux discussions sur l' enseignement en Afrique; le cheminement $d$ ' apprentissage de ce système démontre, en effet, qu' un enseignement arithmétique standardisé peut exister dans une société qui $\mathrm{n}^{\prime}$ a développé aucun système mathématique, qui est à peine alphabétisée, et dans laquelle l' écriture est surtout une technologie dont on a besoin pour la reproduction des textes islamiques et non pas pour l' organisation de la société. Le système des Monts Mandingues - qui est un système avec vingt-deux «cases» - est analytiquement et historiquement une variation du système très connu des seize cases ${ }^{2}$. Ce système de seize cases est utilisé au Mali (et ailleurs) par des marabouts et beaucoup d' études y ont été consacrées, par exemple l' analyse formelle de Jaulin (1966). D' autres auteurs plus récents ont surtout élaboré sur le livre de Jaulin ${ }^{3}$.

2 Les experts en géomancie à Farabako maîtrisent également, plus ou moins, le système de géomancie avec seize cases. Ils estiment cependant que ce système est totalement différent $d u$ leur; chaque système ayant ses règles et interprétations spécifiques ${ }^{4}$. Ils appellent leur propre système kinyèda (kinyè = sable; $d a=$ former, créer, donner forme à quelque chose, exposer) $)^{5}$, et aussi kala jan (littéralement, long bâton). Cependant, ils appellent le système islamique laturu (qui est le terme bamana/maninka pour exprimer la géomancie [Bailleul 1996 : 256]).

\section{À la recherche d' un objet d' étude}

Dans cet article, $\mathrm{j}^{\prime}$ ai choisi de donner la primauté au curriculum du système comme objet de recherche parce que celui-ci est scientifiquement digne de foi (peut être 
reproduit par un chercheur scientifique) et traite d' un aspect de la géomancie qui est rarement au centre de l' attention. $C^{\prime}$ est pourquoi cet article montre surtout comment l' expert géomancien fait son schéma dans le sable ainsi que les comportements, attitudes et opérations qui l' accompagnent. L' accent est mis sur ce que le maître (karamògò) dit et montre à son élève (kalanden).

4 Le fait que ce système de géomancie s' apprenne à l' aide de six leçons arithmétiques bien définies - qui forment l' ossature de l' apprentissage - sera le point principal ${ }^{6}$. Mes expériences en tant qu' élève me permettent de mettre l' accent sur les opérations. Si j' étais toujours le bienvenu lors des sessions de géomancie, mes questions en revanche étaient considérées comme inopportunes; je devais, tout simplement, faire, écouter et apprendre en imitant. Cette règle était pour Namagan et moi-même une forme appréciée d' apprentissage ${ }^{7}$.

5 Cet article porte sur l' apprentissage et décrit la matière sous la forme de six leçons que reçoit un élève s' il se montre intéressé par la géomancie. Dans ces six leçons, l' élève doit apprendre comment un schéma de géomancie de vingt-deux cases doit être dessiné. Dans la description que je donne des six leçons, j' explicite les «cycles d' apprentissage » pour que le lecteur expérimente la force du système éducatif local et apprenne sa logique par des « règles pratiques». Les descriptions faites sont basées sur les dizaines de sessions de géomancie auxquelles j' ai assisté à Farabako, le plus souvent sous la direction de Namagan, qui, malgré son jeune âge relatif, est connu même en dehors de la région comme un expert en géomancie.

\section{Par qui, pour qui ?}

6 «Dieu a créé trois sortes de relations humaines : quand deux méchantes personnes se rencontrent, la relation dure longtemps, quand deux personnes gentilles se rencontrent, la relation dure longtemps; quand une personne méchante et une personne gentille se rencontrent, alors la relation dure trois jours $»^{8}$.

7 Lorsque je visionnais mes enregistrements vidéo des années 2002 et 2003, l' un de mes amis $\mathrm{m}$ ' ayant aidé pour la traduction, le néerlandais d' origine malienne, Boubacar Tamboura, se mit à éclater de rire. La cause était 1 ' explication citée plus haut que Namagan donnait à l' un de ses amis qui venait le consulter. Namagan donna une réponse diplomatique sur la nature de ses pratiques : c' est au visiteur de décider le genre de relation qu' il veut entretenir avec lui. Il peut aider aussi bien quelqu' un qui a de bonnes intentions que quelqu' un qui est malintentionné, mais il ne fera jamais en sorte qu' une personne bien intentionnée ait des problèmes. Car une telle relation ne fait jamais long feu.

Quoique beaucoup de musulmans de Farabako rejetaient les pratiques de mes maittres, ces derniers eux-mêmes se référaient à Dieu comme centre de la géomancie. Namagan me le résumait ainsi : « $C^{\prime}$ est moi qui suis responsable de l' offrande (sarakabaga), mais c' est Dieu qui est responsable de l' acceptation de l' offrande (sarakaminèbaga) ».

On fait presque toujours appel à Dieu lors d' une ou plusieurs phases d' une session de géomancie. Quand un client cherchait, à travers le sable, à savoir si son chef allait un jour lui verser le reliquat de son salaire impayé, Namagan me dit: «Il a perdu tout espoir, que Dieu fasse qu' il le retrouve (A jigi latègèlen, Alla m' a jigi tugun).» Lors de l' 
accomplissement d' un sacrifice, l' expert prononcera également des bénédictions dans lesquelles il demandera l' aide de Dieu : «Fasse Dieu... (Alla ka...).»

Cette perspective tournée vers Dieu, l' expert la connaît dans toutes les phases du sacrifice. En outre, il n' est, naturellement, plus possible de savoir si toutes les phases de la géomancie dans les Monts Mandingues sont d' origine islamique, ou si c' est un système de pratiques non islamiques qui, au fil des années, se sont adaptées aux pratiques et formes islamiques. Étant donné que, lors d' une session, l' expert accorde une attention particulière à ses maîtres, et qu' il fait appel aux " huit esprits et huit personnes » (voir leçon 1), il y a certainement (aussi) des éléments non islamiques dans la géomancie dans les Monts Mandingues.

11 En plus des nombreuses allusions à Dieu, j' ai aussi vu des comportements qui sont à couteaux tirés avec lui ; ce qui explique pourquoi beaucoup de musulmans condamnent la géomancie. Lors des sessions auxquelles $\mathrm{j}$ ' ai assisté en tant qu' "élève de niveau avancé », Namagan demandait souvent non pas à Dieu (Alla), mais à ses basiw (objets de pouvoir) $d^{\prime}$ accepter un sacrifice. Souvent, il s' excusait auprès de ses basiw de ne pouvoir leur offrir qu' une poule et par la suite, jetant des moitiés de noix de cola, il demandait aux basiw s' ils étaient (provisoirement) satisfaits de cette poule. Au cas où les basiw n' étaient pas satisfaits, alors il devait soit présenter des excuses et reprendre la question, soit promettre dans le futur un sacrifice plus grand et redemander ensuite si le basi (sing.) acceptait.

$C^{\prime}$ est donc soit Dieu qui donne souvent les réponses, soit le basi; dans les deux cas, l' expert se sent intermédiaire. Reste à savoir qui vient voir l' expert pour consulter le sable. Les experts consultent tous les jours le sable pour eux, mais pour un client, il faut qu' il y ait une raison spéciale, comme un trouble physique. Ceux qui venaient de loin pour se soigner à Farabako étaient surtout des gens qui avaient ce qu' on peut appeler des troubles chroniques. Après avoir vainement essayé plusieurs années les services de santé biomédicaux occidentaux et $s^{\prime}$ être financièrement ruinées, ces personnes se tournent désormais vers les services gratuits («no cure, no pay») de l' expert à Farabako. Ces gens considèrent souvent leurs infections comme étant du korote, un poison magique qu' on peut donner à quelqu' un à distance. Dans la pratique, cela veut dire que le patient se présente tous les jours chez Namagan ou chez un autre expert pour obtenir une potion ou un conseil. Un tel cocktail de thérapie peut durer plusieurs mois. Pendant ce temps, le patient peut faire quelques activités pratiques bénévoles. Après son retour à la maison, libre à lui de décider combien il doit envoyer à son guérisseur pour le remercier. Namagan $\mathrm{m}$ ' a dit que de cette façon, il a pu acquérir plusieurs vaches.

13 Une autre plainte très fréquente concerne ce qu' on appelle le cèya. $C^{\prime}$ est-à-dire tout ce qui est relatif à la virilité. Cela peut aller de l' impotence - un désagrément contre lequel chaque expert en géomancie a des médicaments - à la peur des esprits de la forêt, un mal contre lequel le service biomédical $n$ ' a pas de solution.

En dehors de ces gens, que l' on peut considérer comme des « déçus » des «services de santé réguliers », Farabako reçoit aussi régulièrement la visite d' habitants de la ville avec toutes sortes de problèmes économiques et sociaux tels que les salaires non payés, le chômage, l' intention d' aller en France ou des problèmes de relations de couples. Ces personnes ont souvent $\mathrm{l}^{\prime}$ impression que $\mathrm{c}^{\prime}$ est $\mathrm{l}^{\prime}$ incompréhension de leurs traditions qui est la cause de leurs problèmes. $C^{\prime}$ est pourquoi ils se rendent « en brousse " pour une consultation. La «brousse » étant le lieu où l' on peut trouver la tradition. Ce 
phénomène est amplement décrit dans les études sur la "modernité " en Afrique subsaharienne (Ashforth 2000 ; Geschiere 2000); on a besoin de «l' occulte » ou de la «tradition » pour trouver un lien avec les choses nouvelles - l' idée « classique » selon laquelle la «modernité » chasse la tradition reste incorrecte.

Namagan Kanté est très actif dans ce "créneau » pour les citadins qui pensent que le fait de perdre la tradition est la raison de leur manque de succès dans le monde moderne. Il a son réseau dans les grandes villes comme Bamako, Kita et Siguiri (en Guinée), des lieux où il séjourne régulièrement ${ }^{9}$.

Pour finir, il est remarquable que Namagan soit très consulté par les Peuls. $C^{\prime}$ est en effet seulement lors des dernières décennies que les Peuls se sont installés dans la région, et il est évident que Namagan est un intermédiaire entre eux et son propre peuple maninka. Namagan a acquis cette position depuis sa jeunesse, lorsqu' il voyageait dans la région avec les vaches de Farabako, ce qui est une activité atypique pour un Maninka (une ethnie de cultivateurs) ${ }^{10}$.

17 Contrairement à son village et à sa famille, Namagan, s' occupant de nouvelles formes de production qui le mettent en contact avec de nouveaux groupes de personnes, satisfait au modèle anthropologique de ce qu' on appelle un «bricoleur » ou un "ethnic broker ». Cependant, lui-même se présente toujours comme un Maninka traditionnel mettant toujours l' accent sur les "normes et valeurs traditionnelles maninka ».

La citation de Namagan évoquée au début de ce chapitre montre qu' il comprend sa connaissance comme une aptitude neutre recevant un contenu moral seulement par l' intention avec laquelle elle est utilisée. Cela s' applique également aux élèves que Namagan reçoit. Ces derniers pouvaient être des écoliers profitant des vacances pour combler leurs lacunes dans le domaine de la "tradition ", mais le plus souvent il s' agissait de jeunes gens qui passaient toute la saison des pluies chez Namagan, qui cherchaient et recueillaient $l^{\prime}$ information auprès de lui, auprès $d$ ' autres jeunes de Farabako et entre eux-mêmes. Eux, je ne les entendais jamais discuter de la géomancie ; chacun pensait que la géomancie pouvait être bonne, mauvaise, islamique et non islamique, et, qu' elle était faite pour tout le monde.

\section{Les six leçons de Namagan Kanté}

La géomancie avec seize différents signes est un système ancien. Chacun des signes représente un code (binaire) spécifique (voir leçon 1). Ces seize signes sont utilisés dans un schéma de seize positions, souvent appelés cases. Ce système de seize signes différents et seize cases est connu dans le monde arabe et une grande partie de l' Afrique.

Les experts en géomancie des Monts Mandingues possèdent les mêmes seize codes que ce système si bien décrit et ils utilisent également seize signes différents. Cependant, le schéma qu' ils utilisent compte vingt-deux cases au lieu de seize. En plus, ces experts ont leurs propres signes pour les codes, et ces signes ont des noms qui ne font pas directement référence à une quelconque source arabe (voir leçon 1), ce qui fait que $\mathrm{l}^{\prime}$ interprétation du schéma de géomancie est spécifique pour cette région.

Dans les Monts Mandingues, la géomancie est enseignée d' une manière qui ne ressemble pas aux méthodes $\mathrm{d}^{\prime}$ enseignement utilisées actuellement dans les écoles fondamentales. $L^{\prime}$ élève apprend beaucoup $d^{\prime}$ opérations pratiques en imitant. Le 
maître corrige les opérations de l' élève, mais il ne donne presque jamais $\mathrm{d}^{\prime}$ instructions explicites. L' élève ne doit jamais demander directement pourquoi telle chose se passe de telle façon. La plupart du temps, il est occupé à exécuter, pour son maître, des tâches diverses, comme aller chercher des plantes médicinales en brousse et les faire bouillir. En plus, il doit travailler dans le champ du maître, faire des petits travaux et lui offrir de temps à autre un petit cadeau.

Un maître donnera des leçons à son élève seulement quand il sera satisfait du comportement de ce dernier. Moi, je devais par exemple attendre plusieurs mois avant de recevoir les leçons 1 et $2^{11}$.

Grâce à son accessibilité, la description suivante des leçons de Namagan est différente des leçons elles-mêmes. Étant donné qu' on n' a plus besoin d' être patient, le lecteur peut arriver à un niveau pour lequel un élève autochtone devra attendre plusieurs mois.

Celui qui prendra la peine d' étudier les six leçons découvrira qu' elles comportent une trajectoire didactique. Chaque leçon se construit sur la base de la précédente et, au fur et à mesure, leur complexité augmente. De plus, à chaque leçon, l' élève apprend, implicitement, certaines règles pratiques. De cette façon, les règles favorisent la pensée logique et l' intelligence arithmétique. Le lien mutuel entre les leçons est tellement grand qu' au bout de six exercices l' élève aura un aperçu global des aspects arithmétiques d' un schéma de géomancie. Il y a, en outre, des points d' appui dans les leçons qui réconfortent $\mathrm{l}^{\prime}$ élève dans le processus $\mathrm{d}^{\prime}$ apprentissage parce qu' il s' aperçoit qu' il maîtrise déjà certains aspects.

\section{Leçon 1 : Les « huit esprits et huit personnes »}

La première leçon (kalan folò) a pour but de montrer à l' élève qu' il se cache un code binaire derrière chacun des seize signes de la géomancie. Avec cette première leçon, l' élève apprend à transcrire, de droite à gauche, les deux rangées suivantes de huit codes chacune (voir fig. 1).

Pour les codes, on met ses doigts dans le sable en faisant, de haut en bas, des traits de 5 $\mathrm{cm}$ environ. On fait un trait unique avec l' index, un trait double avec l' index et le majeur. Ceci donne la figure suivante (j' ai utilisé la lettre I pour indiquer un trait vertical) :

$\begin{array}{llllllll}\text { I } & \text { I } & \text { I } & \text { I } & \text { II } & \text { II } & \text { II } & \text { II } \\ \text { I } & \text { I } & \text { II } & \text { II } & \text { I } & \text { II } & \text { I } & \text { II } \\ \text { I } & \text { II } & \text { I } & \text { II } & \text { I } & \text { I } & \text { II } & \text { II } \\ \text { I } & \text { I } & \text { I } & \text { I } & \text { II } & \text { II } & \text { II } & \text { II } \\ & & & & & & & \\ \text { II } & \text { I } & \text { II } & \text { I } & \text { II } & \text { I } & \text { II } & \text { I } \\ \text { I } & \text { I } & \text { II } & \text { I } & \text { I } & \text { II } & \text { II } & \text { II } \\ \text { I } & \text { I } & \text { I } & \text { II } & \text { II } & \text { I } & \text { II } & \text { II } \\ \text { I } & \text { II } & \text { I } & \text { II } & \text { I } & \text { II } & \text { I } & \text { II }\end{array}$

Fig. 1. - Seize codes de la géomancie des Monts Mandingues les « huit esprits et huit personnes » (à liRe de droite À GAUCHE)

27 Le lecteur doué en statistiques verra là les seize codes binaires qu' on obtient quand on fait un « bit » de quatre $0 / 1$ combinaisons; il y a $2 \times 2 \times 2 \times 2=16$ combinaisons $^{12}$. 

signes dans un seul mouvement avec l' index et le majeur (de même pour nimisa); quand il s' agit d' une seule ligne, on la trace avec l' index. Pour le jubidibinè, kumadibinè et garela(n), on utilise également l' annulaire. De plus, le bout par lequel on commence à tracer un signe est très important. On écrit de droite à gauche et de haut en bas. Au début, $\mathrm{j}$ ' écrivais les signes nyagaransé et tèrèmèsè en faisant un trait de gauche à droite - ce qui me paraissait normal étant donné qu' à l' école j' ai appris à écrire de cette façon -, mais Namagan me demandait de les reprendre : il faut que ce soit de droite à gauche!

interprétation des signes et des combinaisons de signes est pour moi une terra incognita. Mais on peut dire que les noms des signes $\mathrm{n}^{\prime}$ ont pas de significations importantes pour l' interprétation; Namagan lui-même ne pouvait trouver aucune signification (kòrò) au nom (tògò). Nimisa veut dire regret dans la vie courante, mais cela $\mathrm{n}^{\prime}$ a certainement aucune conséquence pour l' interprétation. McNaughton (1988:54) dit que « Kumardisé» « l' homme noir » est un «Yarase» «les femmes noires ». " Maro maro " serait l' enfant dans le ventre de sa mère. Derive et Dumestre (1999: 34) appellent "Dyouroubassé » ("Jùrrubase » ou "Jùubase») le nom du frère jumeau de Mande Mori, le héros chasseur ${ }^{16}$. Selon ses auteurs, «juru » ferait allusion aux cordes d' un instrument de musique. Le dictionnaire (Bailleul 1996: 395) donne quelques mots 
disant peut-être quelque chose sur les différentes composantes dans les noms : «tere » ou «tèrè » veut dire " hasard, chance, sort », "tèrema » veut dire "quelqu' un qui a un revers de fortune » et « tèrèmè » fait allusion à la négociation d' un prix. «Tamana » veut dire «flamme ", « kuma» signifie «mot/parole » et «dibi» signifie « obscurité ». Bien que des notions telles que "parole» et «obscurité » soient importantes dans la cosmologie des Maninka (Jansen 2002: 42) ${ }^{17}$, les informateurs locaux $n^{\prime}$ utilisaient presque jamais ces termes quand je leur demandais la signification des noms des seize signes.

Je n' ai jamais entendu les géomanciens discuter ou spéculer sur les significations des noms des signes. Dans la région de la Sénégambie, Graw (2005) a entendu, auprès des experts géomanciens d' origines ethniques différentes, toutes sortes de termes ayant des liens linguistiques et des sons avec les noms que j' entendais dans les Monts Mandingues. $C^{\prime}$ est pourquoi je pense que la géomancie dans ce qu' on appelle le « monde mandé » a (en partie) un jargon partagé qui acquiert une signification dans les pratiques locales.

Les huit esprits et huit personnes doivent être tracés dans un ordre bien déterminé. Cela m' est apparu clairement avec la réaction de Namagan quand, après un long séjour aux Pays-Bas, j' ai décidé d' utiliser mon propre ordre lors de la répétition de la leçon 1 ; Namagan $\mathrm{m}^{\prime}$ arrêtait et je devais recommencer. Paulus Gerdes ${ }^{18} \mathrm{~m}$ ' a fait remarquer que la figure de deux fois huit colonnes se caractérise par différentes symétries (de rotation). Selon Gerdes, celles-ci peuvent être importantes, par exemple comme aidemémoire.

Sur ce, nous arrivons à la fin de la première leçon dans laquelle l' élève aura appris que derrière chacun des seize signes de géomancie se cache un code binaire. De plus il aura appris comment tracer les signes dans le sable et quels noms portent les signes.

\section{Leçon 2 : Quatre jubidisè}

La leçon 2 (kalan filanan, littéralement deuxième leçon) consiste à reproduire le schéma suivant de vingt-deux signes. Le maître ne donne aucune explication complémentaire ; il fait les signes et donne leurs noms. L' élève reçoit l' ordre d' apprendre ce schéma :

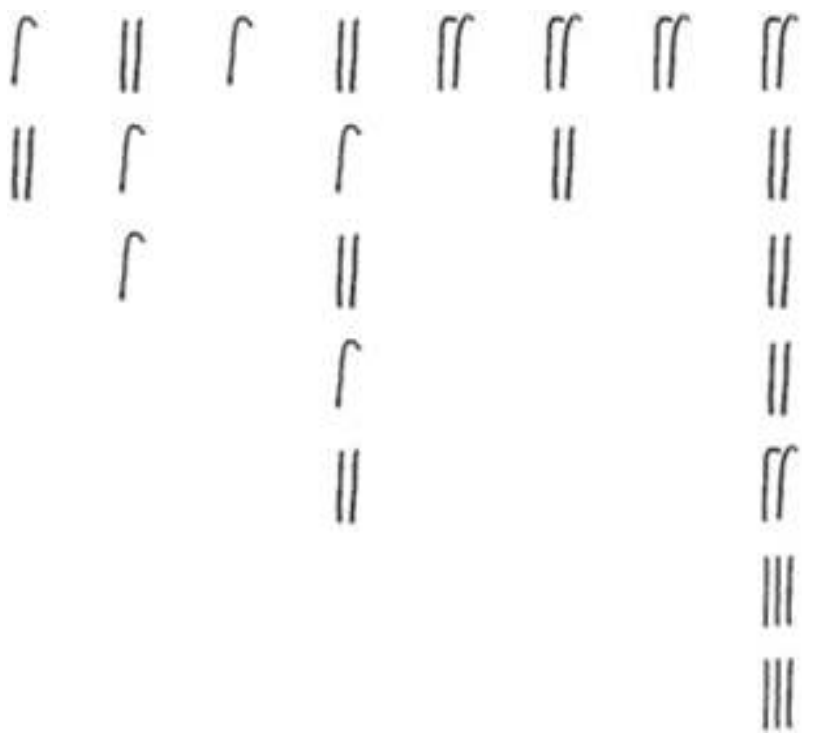

FIG. 3. - Le SCHÉMA dE LA LEÇON 2 


\begin{tabular}{|l|l|l|}
\hline Pair & +Pair & = Pair \\
\hline Impair & +Impair & $=$ Pair \\
\hline Pair & +Impair & $=$ Impair \\
\hline Impair & +Pair & =Impair \\
\hline
\end{tabular}
« leçon 2 », tous les quatre signes de base sont des jubidisè. $C^{\prime}$ est pourquoi le lecteur reconnaîtra facilement le schéma suivant :

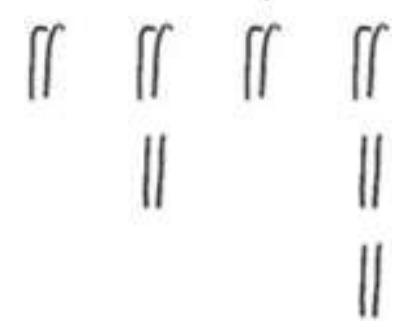

Fig. 5. - Les SEPT PREMIERS SIGNES DE LA LEÇON 2

Les troisième et quatrième signes initiaux donnent également un siké comme résultat. Quand ils sont regroupés, les deux siké de la deuxième rangée donnent un siké dans la troisième rangée. Comme on peut s' y attendre, celui-ci sera tracé dans la colonne à l' extrême droite. 
Pour reproduire les quatre prochains signes, 1 ' élève doit se rappeler les quatre signes initiaux comme des codes binaires (verticaux) pour ensuite les lire de façon horizontale. Il met ainsi la rangée d' en haut, comme colonne, à gauche des quatre signes initiaux et la deuxième rangée à gauche de ces derniers. En code binaire, cela donne l' image suivante :

$\begin{array}{llllll}\text { I } & \text { II } & \text { II } & \text { II } & \text { II } & \text { II } \\ \text { I } & \text { II } & \text { I } & \text { I } & \text { I } & \text { I } \\ \text { I } & \text { II } & \text { II } & \text { II } & \text { II } & \text { II } \\ \text { I } & \text { II } & \text { I } & \text { I } & \text { I } & \text { I }\end{array}$

Fig. 6. - Les Codes des signes initiauX et Les deuX premières transformations

On voit donc que la rangée d' en haut des quatre signes initiaux sont quatre II (cursif et en gras) et cela donne siké (cursif dans la fig. 6). En traçant ce signe, le maître géomancien ne prononce pas seulement le nom du signe, mais aussi, au préalable, le terme « kinyèkun » ( la tête du sable »).

48 La deuxième rangée des quatre premiers signes initiaux de la leçon 2 (quatre jubidisè dans ce cas) consiste seulement en un I et cela donne tamanakaté. Alors, on peut ajouter les trois prochains signes (siké et tamanakaté donnent toujours un tamanakaté) :

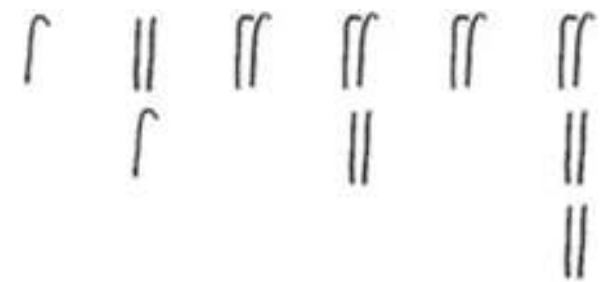

Fig. 7. - LA transformation des Signes INITIAUX, PREMIÈre ÉTAPE

Encore une fois, l' élève dispose ici d' une règle de base. Il voit qu' en ajoutant un signe à un siké, cela ne change pas le signe en question; dans le cas précis, le tamanakaté reste inchangé.

L' élève doit ensuite lire "horizontalement » les troisième et quatrième rangées des signes initiaux. Cela donne encore une fois un siké et un tamanakaté. Regroupés, les deux forment ensemble un tamanakaté. Le résultat est le suivant :<smiles>C=C=C=C=C=C</smiles>

Fig. 8. - LA tRANSFormation des SIGNES INITIAUX, DEUXIÈME ÉTAPE

51 Les deux prochains signes sont maintenant faciles à comprendre; deux tamanakaté donnent un siké et deux siké donnent un siké: 


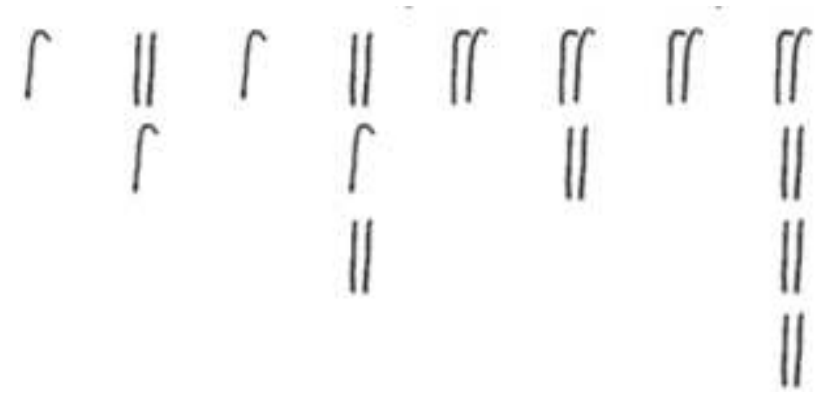

FIG. 9. - LeS PREMIERS QUINZE SIGNES DE LA LEÇON 2

Un tel schéma de quinze cases à partir de quatre codes initiaux est un principe qui est déjà connu depuis des siècles, dans plusieurs parties du monde ; $c^{\prime}$ est en fait aussi la base du système bien répandu des seize cases. Dans ce système, on reçoit un seizième et dernier signe en regroupant le quinzième signe au premier signe initial (le signe d' en haut à droite). Il y a eu au fil des années beaucoup d' études sur ce système de seize cases.

Cependant, les experts géomanciens des Monts Mandingues continuent d' une autre façon, qui, selon moi, est unique et n' a jamais été décrite.

Le système des Monts Mandingues est, « selon la technique de calcul», plus difficile que le système plus connu de seize cases. Le plus difficile, dans les leçons de géomancie dans les Monts Mandingues, est justement la partie qui suit.

Afin de comprendre la formation des sept derniers signes, l' élève doit savoir dans quel ordre préétabli le maître forme les signes; ce dernier n' accepte pas qu' on s' écarte de cette procédure. $C^{\prime}$ est pourquoi, afin d' appuyer la suite de ma description, je donne d' abord le schéma dans lequel je numérote les signes par ordre de production.

Les experts eux-mêmes ne pensent pas en termes de positions avec un numéro. Ils appellent les positions des «cases» («sow») et donnent, au cours de leur interprétation, des caractéristiques spéciales à certaines des cases (voir ci-dessus).

57 Cependant, ce schéma avec l' ordre de succession des positions est nécessaire au lecteur pour qu' il comprenne la façon de tracer le schéma de géomancie. Le schéma avec l' ordre de succession des positions se présente comme suit :

\begin{tabular}{|c|c|c|c|c|c|c|}
\hline & & & \multirow{2}{*}{$\begin{array}{r}8 \\
10\end{array}$} & \multirow{2}{*}{5} & \multirow{2}{*}{$\begin{array}{l}4 \\
6\end{array}$} & \multirow[t]{2}{*}{2} \\
\hline & & & & & & \\
\hline & $\begin{array}{l}12 \quad 11 \\
13\end{array}$ & 9 & \multicolumn{2}{|l|}{14} & 6 & \\
\hline
\end{tabular}

FIG. 10. - L' oRdRE dE GÉNÉRATION DES QUINZE PREMIÈRES CASES/POSITIONS

La formation des seizième, dix-septième et dix-huitième signes est différente en ce sens qu' ici, on ne fait pas de regroupement de signes (qui sont proches dans le schéma) les uns avec les autres. Pour faire le seizième signe, l' expert ajoute le signe dans la position 5 (le quatrième signe initial) ensemble avec le signe à la position 15 (le signe situé le plus en bas, la fin du «schéma de base »). Il fait de même pour obtenir le dixseptième signe. Pour ce faire, il ajoute la position 12 à la position 14. Les deux nouveaux signes, regroupés, produisent le dix-huitième signe (position $18=$ position $16+$ position 17). Le schéma de la leçon 2 ressemble à ce qui suit : 


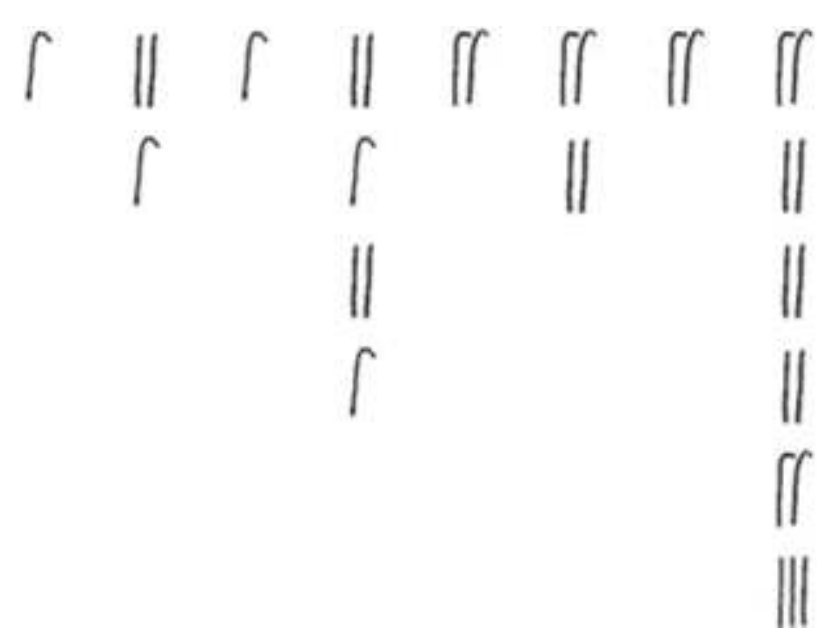

FIG. 11. - LES DIX-HUIT PREMIERS SIGNES DE LA LEÇON 2

59 Ce point est également une étape qui constitue une règle de base : en ajoutant tamanakaté à un autre signe, on obtient un " reflet » de ce signe (pour savoir pourquoi je choisis cette métaphore du miroir, voir fig. 1 dans la leçon 1). Celui qui étudie à fond la géomancie s' apercevra qu' on peut apprendre facilement le regroupement d' un tamanakaté avec un autre signe grâce à «l' effet de miroir ». C' était apparemment le cas aussi pour les élèves de Namagan, car eux aussi effectuaient relativement vite, sans faute et sans hésitation, le regroupement d' un signe avec tamanakaté.

Quand on apprend bien les signes et leurs codes, on peut facilement assimiler la formation des dix-huit premiers signes. La production du signe à la position 19 est si complexe qu' il constitue, aussi bien pour l' élève que pour nous, une grosse pierre d' achoppement. Celui qui est capable d' accomplir, de mémoire et sans faute, le dixneuvième signe en dix secondes peut se considérer comme un expert !

61 Le « calcul» du signe à la position 19 exige une conversion (imaginaire) de la rangée supérieure de signes en codes binaires. En ce qui concerne la leçon 2, cette rangée se présente comme suit :

$\begin{array}{llllllll}\text { I } & \text { II } & \text { I } & \text { II } & \text { II } & \text { II } & \text { II } & \text { II } \\ \text { I } & \text { II } & \text { I } & \text { II } & \text { I } & \text { I } & \text { I } & \text { I } \\ \text { I } & \text { II } & \text { I } & \text { II } & \text { II } & \text { II } & \text { II } & \text { II } \\ \text { I } & \text { II } & \text { I } & \text { II } & \text { I } & \text { I } & \text { I } & \text { I }\end{array}$

Fig. 12. - LA RANGÉE SUPÉRIEURE DE LA LEÇON 2, PRÉSENTÉE EN CODES BINAIRES

62 Regardez maintenant la rangée du bas de cette figure et, lisant de droite à gauche, prenez le « bit » du bas des deuxième, quatrième, sixième et huitième colonnes (dans la fig. 12, ces derniers sont cursifs et en gras). Dans le cas présent, cela veut dire quatre fois I (impair) et donne un signe qui est, ici, un tamanakaté. On ajoute ce tamanakaté « imaginaire » au signe à l' extrême gauche (le signe à la position 12, dans ce cas, lui aussi est un tamanakaté), et l' on obtient de cette façon le signe à la position 19. Ce signe se trouve sous la position 12. Dans notre exemple le signe sous la position 19 est un siké.

On s' attend à ce que $\mathrm{l}^{\prime}$ élève déduise lui-même cette procédure à partir des actions du maître. $C^{\prime}$ est peut-être pourquoi Namagan était peu empressé quand il $\mathrm{m}^{\prime}$ expliquait, en novembre 1999, le principe sous-jacent. Je suppose que normalement, un élève est censé apprendre cela avec un de ses collègues. 
64 En faisant le signe à la position 19, le maître montre respectivement les signes aux positions 2, 5, 9 et 12 pendant qu' il prononce les mots suivants : « Ka wuli [nom du signe à la position 2], $k a$ na [nom du signe à la position 5], $k a$ se/si [nom du signe à la position 9]. [Nom du signe imaginaire]-ba bè [nom du signe à la position 12] sòrò ka ko fila [nom du signe à la position 19] $»^{20}$.

65 Dans la leçon 2, cela signifie: "Ka wuli jubidisè, ka na jubidisè, ka se tamanakaté. Tamanakatéba bè tamanakaté sòrò ka ko fila siké» ("Quitter jubidisè, venir à jubidisè, arriver à tamanakaté. Un grand tamanakaté trouve un tamanakaté et les deux forment un siké »).

Les trois derniers signes se forment ensuite $d^{\prime}$ une manière qui peut paraître connue au lecteur. À la position 20 vient le regroupement des signes aux positions 13 et 19. La position 21 est le résultat du regroupement des positions 17 et 20 . Le dernier signe est obtenu en ajoutant les positions 18 et 21.

67 L' élève devra, par la suite, toujours remplir les vingt-deux positions selon cet ordre spécifique ; le maître n' acceptera pas qu' on s' écarte de cet ordre :

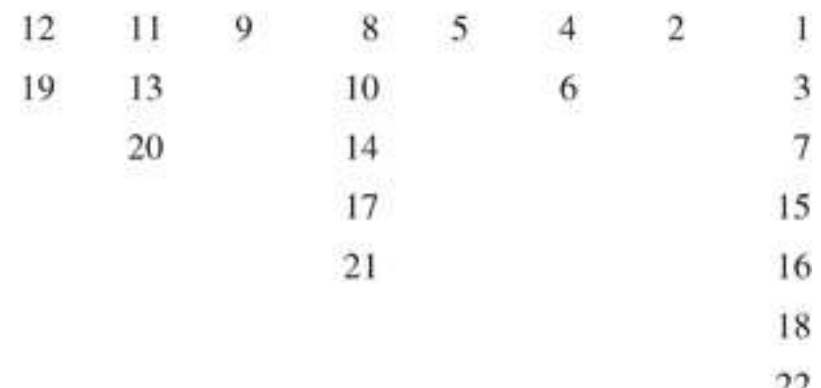

FIG. 13. - L' ORDRE DE GÉNÉRATION DES VINGT-DEUX SIGNES

Le schéma de la génération elle-même est :

\begin{tabular}{|c|c|c|c|c|c|}
\hline 12 & 11 & 9 & 8 & 4 & 1 \\
\hline $\begin{array}{l}19 \\
\text { (voir texte) }\end{array}$ & $\begin{array}{l}13 \\
(=11+12)\end{array}$ & & $\begin{array}{l}10 \\
(=8+9)\end{array}$ & $\begin{array}{l}6 \\
(=4+5)\end{array}$ & $\begin{array}{l}3 \\
(=1+2)\end{array}$ \\
\hline & $\begin{array}{l}20 \\
(=13+19)\end{array}$ & & $\begin{array}{l}14 \\
(=10+13)\end{array}$ & & $\begin{array}{l}7 \\
(=3+6)\end{array}$ \\
\hline & & & $\begin{array}{l}17 \\
(=12+14)\end{array}$ & & $\begin{array}{l}15 \\
(=7+14)\end{array}$ \\
\hline & & & $\begin{array}{l}21 \\
(=17+20)\end{array}$ & & $\begin{array}{l}16 \\
(=5+15)\end{array}$ \\
\hline & & & & & $\begin{array}{l}18 \\
(=16+17)\end{array}$ \\
\hline & & & & & $\begin{array}{l}22 \\
(=18+21)\end{array}$ \\
\hline
\end{tabular}

Fig. 14. - Le schéma de génération d' un schéma de géomancie dans les Monts Mandingues

Pour ceux qui veulent vérifier leurs propres calculs, il est très facile de reproduire ce schéma dans Excel (voir figure 15) ${ }^{21}$. Les codes que cela donne sont toujours des colonnes de quatre au total (pair et/ou impair) des chiffres (par exemple 4-3-6-0 pour nimisa ou 3-5-7-5 pour tamanakatè). 


\begin{tabular}{|c|c|c|c|c|c|c|}
\hline $\mathrm{H}$ & G & $\mathrm{F}$ & $\mathrm{E}$ & D & $\mathrm{C}$ & $\mathrm{A}$ \\
\hline $1=\mathrm{A} 4$ & $=\mathrm{A} 3$ & $=\mathrm{A} 2$ & $=\mathrm{Al}$ & D1 & $\mathrm{Cl}$ & Al \\
\hline $2=B 4$ & $=\mathrm{B} 3$ & $=\mathrm{B} 2$ & $=\mathrm{B} 1$ & D2 & $\mathrm{C} 2$ & $\mathrm{~A} 2$ \\
\hline $3=C 4$ & $=\mathrm{C} 3$ & $=\mathrm{C} 2$ & $=\mathrm{C} 1$ & D3 & $\mathrm{C} 3$ & A 3 \\
\hline $4=\mathrm{D} 4$ & $=\mathrm{D} 3$ & $=\mathrm{D} 2$ & $=\mathrm{D} 1$ & D4 & $\mathrm{C} 4$ & A4 \\
\hline $5=\mathrm{B} 4+\mathrm{H} 1$ & $=\mathrm{Gl}+\mathrm{HI}$ & & $=\mathrm{E} 1+\mathrm{F} 1$ & & $=\mathrm{Cl}+\mathrm{Dl}$ & $=\mathrm{Al}+\mathrm{Bl}$ \\
\hline $5=\mathrm{D} 4+\mathrm{H} 2$ & $=\mathrm{G} 2+\mathrm{H} 2$ & & $=\mathrm{E} 2+\mathrm{F} 2$ & & $=\mathrm{C} 2+\mathrm{D} 2$ & $=\mathrm{A} 2+\mathrm{B} 2$ \\
\hline $7=\mathrm{F} 4+\mathrm{H} 3$ & $=\mathrm{G} 3+\mathrm{H} 3$ & & $=\mathrm{E} 3+\mathrm{F} 3$ & & $=\mathrm{C} 3+\mathrm{D} 3$ & $=\mathrm{A} 3+\mathrm{B} 3$ \\
\hline $8=\mathrm{H} 4+\mathrm{H} 4$ & $=\mathrm{G} 4+\mathrm{H} 4$ & & $=\mathrm{E} 4+\mathrm{F} 4$ & & $=\mathrm{C} 4+\mathrm{D} 4$ & $=\mathrm{A} 4+\mathrm{B} 4$ \\
\hline 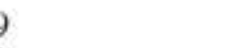 & $=\mathrm{G} 5+\mathrm{H} 5$ & & $=\mathrm{E} 5+\mathrm{G} 5$ & & & $=\mathrm{A} 5+\mathrm{C} 5$ \\
\hline & $=\mathrm{G} 6+\mathrm{H} 6$ & & $=\mathrm{E} 6+\mathrm{G} 6$ & & & $=\mathrm{A} 6+\mathrm{C} 6$ \\
\hline & $=\mathrm{G} 7+\mathrm{H} 7$ & & $=\mathrm{E} 7+\mathrm{G} 7$ & & & $=\mathrm{A} 7+\mathrm{C} 7$ \\
\hline & $=\mathrm{G} 8+\mathrm{H} 8$ & & $=\mathrm{E} 8+\mathrm{G} 8$ & & & $=\mathrm{A} 8+\mathrm{C} 8$ \\
\hline
\end{tabular}

[Image non convertie]

FIG. 15. - SCHÉMA DE GÉOMANCIE DANS EXCEL (À LIRE DE DROITE À GAUCHE)

71 Qu' avons-nous appris dans la leçon 2 ? Le schéma de la leçon 2 (fig. 3) est à juste titre une leçon pour les débutants : elle est simple mais contient des règles de "calcul » cruciales. Le schéma est relativement facile parce que l' on $n^{\prime} \mathrm{y}$ retrouve que quatre signes et que les mêmes paires de signes reviennent toujours. En plus, les combinaisons de la leçon 2 montrent trois règles de calcul très importantes :

72 - quand on regroupe deux signes similaires, le résultat est un siké;

73 - en regroupant un tamanakaté avec un autre signe, on obtient un signe " reflété »;

74 - ajouter un siké « ne change rien » (a tè foyi yèlèma).

75 Ces règles de calcul soutiennent mon argument selon lequel les six leçons forment ensemble un cours. Cela explique pourquoi les élèves peuvent comprendre les leçons sans explications détaillées ${ }^{22}$.

\section{Leçon 3 : Deux siké et deux tamanakaté}

La leçon 3 (kalan sabanan, littéralement la troisième leçon) est facile à suivre pour celui qui maîtrise la leçon 2. La raison en est que la leçon 3 commence avec deux siké et deux tamanakaté comme signes initiaux. Ceci doit être lu de façon «horizontale» ( (inversée ») comme quatre nyagaransè. Ainsi, il n' est pas difficile de faire les dix-sept premiers signes dans le schéma des vingt-deux signes, même pour un débutant :

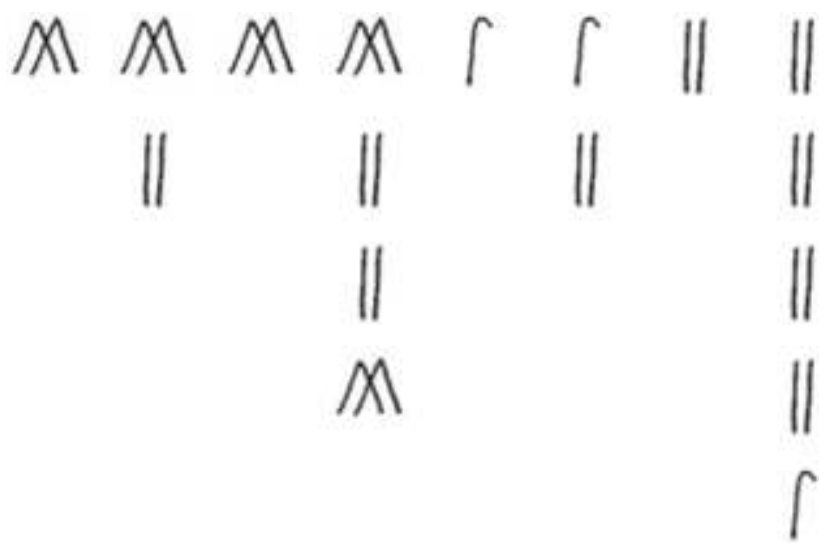

FIG. 16. - LES SCHÉMAS DE LA LEÇON 3 (LES DIX-SEPT PREMIERS SIGNES) 
Cependant, cette leçon est plus difficile que la leçon 2 car il faut remplir les cinq positions restantes ; 1 ' élève apprend, ici, un certain nombre de nouvelles combinaisons qui sont plus difficiles que de "refléter " un signe en l' ajoutant à un tamanakaté (voir leçon 2). $C^{\prime}$ est surtout les cinq dernières positions de la leçon 3 qui demandent beaucoup d' effort à l' élève.

Les moments cruciaux de la leçon 3 se trouvent à la fin de cette leçon; tout d' abord, cette leçon donne à l' élève beaucoup de confiance en soi et, à la fin, le met même au défi de se surpasser. Le résultat final est (voir fig. 17) :

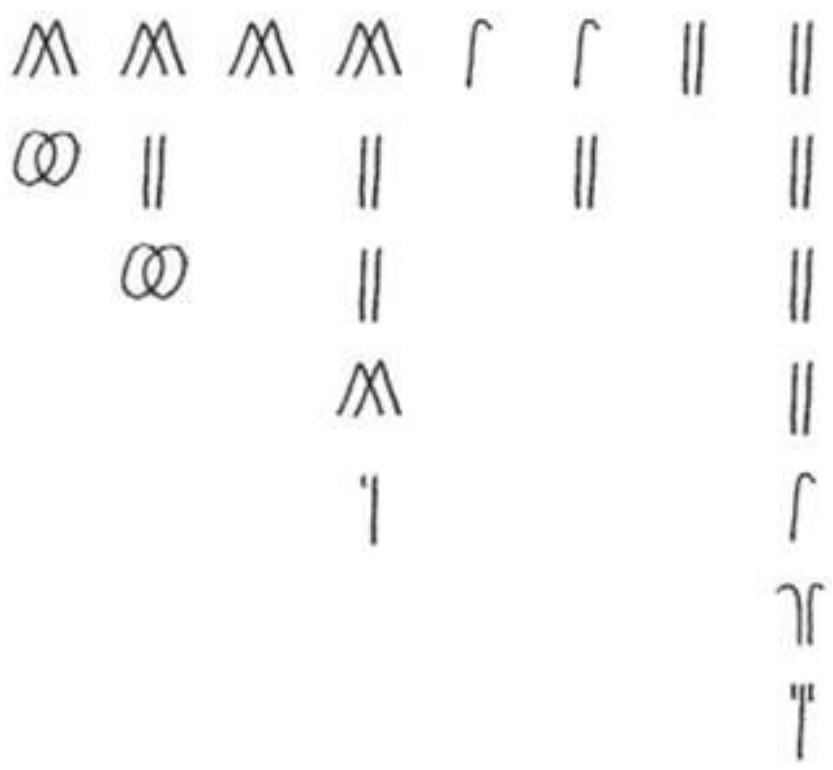

FIG. 17. - LE SCHÉMA COMPLET DE LA LEÇON 3

79 On voit pourquoi cette leçon est plus difficile que la leçon 2 : finalement, elle comporte sept signes différents. L' élève sera surpris par une telle variation «brusque » de «calcul».

\section{Leçon 4 : Un nimisa et trois kumadisè}

La leçon 4 (kalan naaninan, littéralement la quatrième leçon) est plus difficile que les leçons précédentes. Cette leçon comporte onze signes différents. Certains d' entre eux apparaissent plusieurs fois dans le schéma des vingt-deux signes, mais proviennent $\mathrm{d}$ ' un regroupement des deux signes différents. Ainsi, le jubidisè à la position 3 (voir fig. 18) est le résultat du regroupement des signes initiaux nimisa et kumadisè, mais le jubidisè à la position 21, est le résultat du regroupement de maramaro et kumadibinè. La compréhension du fait qu' un signe peut être le résultat de plusieurs combinaisons est une étape importante dans l' apprentissage. 


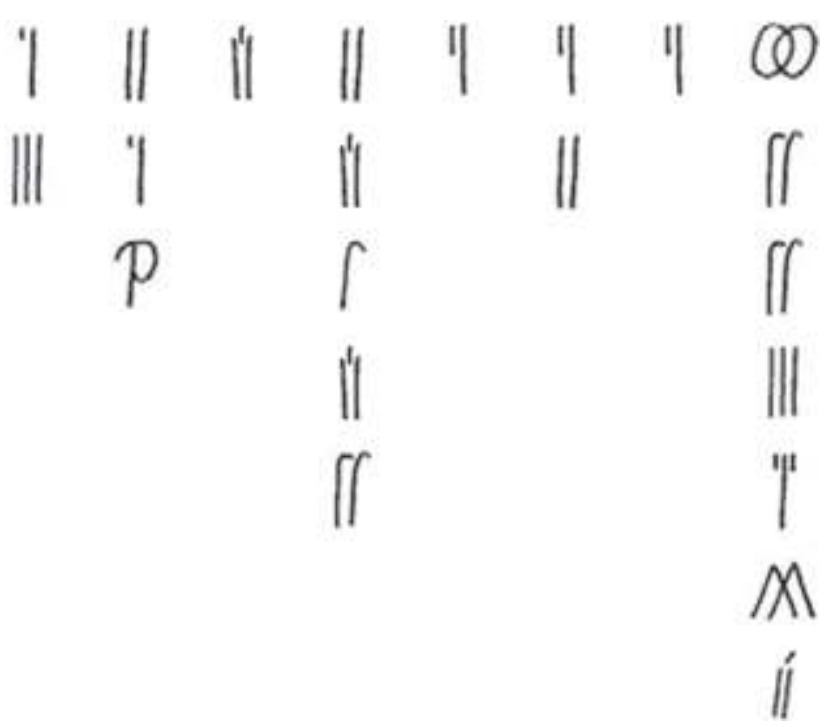

FIG. 18. - LE SCHÉMA DE LA LEÇON 4

81 Cette leçon nous apprend, de façon abstraite, qu' il y a plusieurs combinaisons possibles et qu' un même signe peut être le résultat $d^{\prime}$ un regroupement de plusieurs paires de signes. L' élève talentueux comprend déjà qu' il y a $16 \times 16=256$ combinaisons (ou du moins sentir qu' il y a plusieurs sortes de combinaisons) et que chaque signe peut donc être le résultat de seize combinaisons.

Un autre moment-clé dans cette leçon est que ce schéma apparaît aussi en réalité dans la pratique parce qu' il s' agit de l' un des 256 schémas initiaux du type de géomancie dont il est question ici. Ces schémas initiaux nécessitent quelques explications. Pendant que dans le système plus connu de seize signes possibles et quatre signes initiaux, on travaille avec $16 \times 16 \times 16 \times 16=66536$ schémas initiaux possibles (Jaulin 1966), les experts des Monts Mandingues prennent seulement les signes siké, nimisa, kumadisè et jubidisè comme signes initiaux. Ainsi, il y a seulement $4 \times 4 \times 4 \times 4=256$ schémas initiaux possibles.

Ci-dessous je décris comment sont générés les quatre signes initiaux et quelles conséquences arithmétiques cela comporte ${ }^{23}$.

\section{Leçon 5 : Jubidisè, nimisa, siké et nimisa}

84 La leçon 5 (kalan duurunan, littéralement la cinquième leçon) est, tout comme la précédente, un schéma qui se produit dans la pratique. Dans cette leçon, l' élève comprendra qu' avec un schéma initial, les sept premières positions ont toujours les mêmes quatre signes: siké, nimisa, kumadisè et jubidisè, par addition, se génèrent toujours. L' élève apprend en cela une aptitude qui, dans la pratique, lui servira de base éloquente. Cette règle de base est un moment didactique important parce que l' élève peut, désormais, réaliser les premiers signes en toute confiance.

De plus, il apprend qu' aux positions 8 et 11, il y a toujours un siké (voir la leçon 6 pour l' explication). Cette leçon se présente comme suit : 


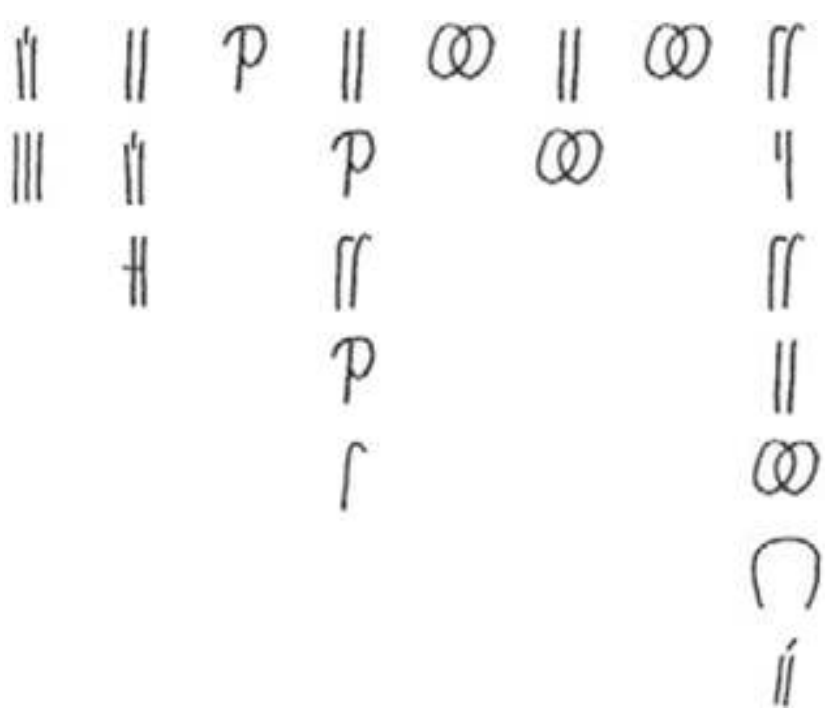

Fig. 19. - Le SCHÉMA DE LA LEÇON 5

\section{Leçon 6 : deuxième étape} initiaux potentiels :

$\begin{array}{llll}\text { II } & \text { II } & \text { II } & \text { II } \\ \text { I } & \text { II } & \text { I } & \text { II } \\ \text { II } & \text { II } & \text { II } & \text { II } \\ \text { I } & \text { I } & \text { II } & \text { II }\end{array}$

Fig. 20. - Les CODES DES QUATRE SIGNES INITIAUX POTENTIELS 
Quels que soient l' ordre de succession et le nombre de signes qu' il y a dans les quatre signes initiaux, il y aura toujours, "lu horizontalement », deux signes siké (en gras et cursif dans la fig. 20). Ces deux siké apparaissent aux positions 8 et 11. Ajoutés respectivement aux positions 9 et 12, les produits des positions 10 (regroupement de 8 et 9) et 13 (regroupement de 11 et 12) seront toujours identiques respectivement aux positions 9 et 12. On voit ce principe dans les leçons 2 (fig. 3), 4 (fig. 18) et 5 (fig. 19) parce que dans ces leçons, les quatre signes initiaux proviennent de «l' union » de siké, nimisa, jubidisè et kumadisè.

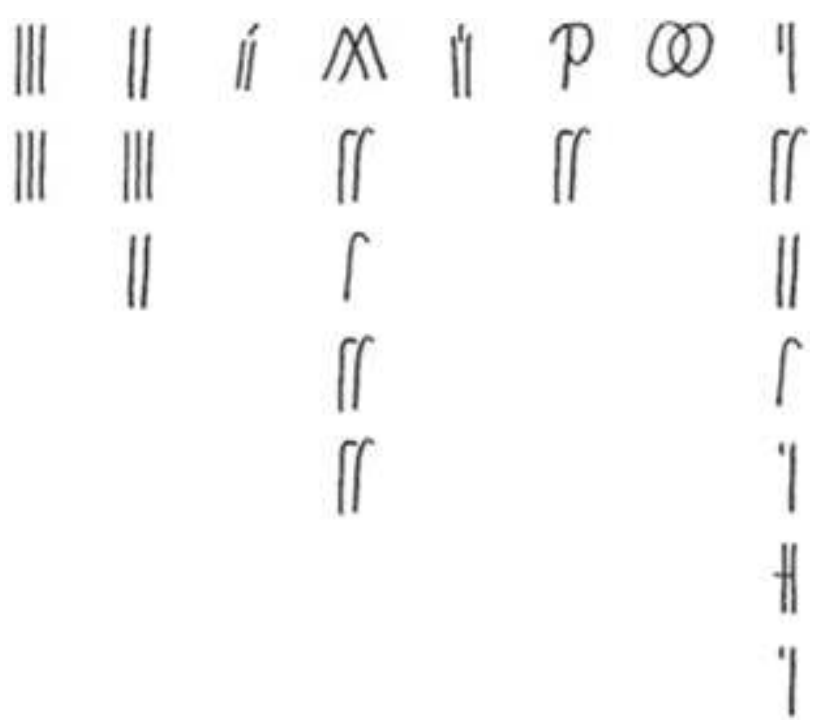

FIG. 21. - LE SCHÉMA DE LA LEÇON 6 ; DEUXIÈME ÉTAPE DE LA LEÇON 5

Je trouve que le choix de quatre signes à partir de la deuxième rangée comme base d' une deuxième étape est un « mécanisme de sécurité » dans le système de géomancie des Monts Mandingues. Ce mécanisme de sécurité limite les fautes lors la formation de la deuxième étape.

Il est ingénieux d' utiliser les signes produits relativement tôt pour une deuxième étape. Les chances que ceux-ci soient le résultat $d^{\prime}$ une erreur de calcul sont relativement minimes parce que les treize premiers signes $d$ ' une première session sont plus faciles à générer que les neuf suivants. Il serait dangereux de prendre, par exemple, les quatre derniers signes étant donné que la plupart des fautes sont commises en formant la position $19^{24}$.

$C^{\prime}$ est avec le schéma de la figure 21 (voir page précédente) que se terminent les kalan woro ("six leçons ») que les élèves reçoivent à Farabako. On peut remarquer qu' une étape ultérieure ne doit évidemment pas conduire à un schéma compliqué ${ }^{25}$. Cela s' avère surtout quand un élève assidu essaie de passer aux prochaines étapes à partir de la leçon 6 (voir fig. 22). La cinquième étape (à partir de la leçon 5 comme schéma de base) apparaît ici comme un "terminus", parce qu' elle génère les mêmes quatre signes successifs que la quatrième étape (un tamanakaté et trois siké); de ce fait, une sixième étape aurait été identique à la cinquième étape. 
Troisième étape

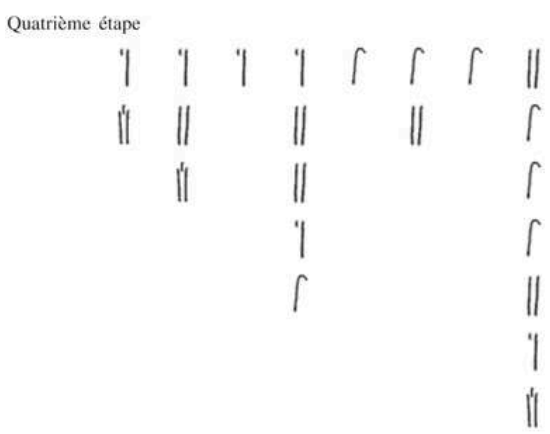

Cinquième étape

$\begin{array}{llllllll}\| & \| & \| & \| & \| & \| & \| & \uparrow \\ \| & \| & & \| & & \| & & \end{array}$

95 [Image non convertie]

Fig. 22. - Schémas qui APPARAisSent EN CONTINUANT AVEC LA LEÇON 6

\section{Présentation au sable et détermination des quatre signes initiaux}

L' expert consulte généralement le sable dans sa propre case, là où ses basiw (objets de pouvoir) se trouvent. Cependant, tout autre lieu est possible. L' expert commence une session en s' asseyant à califourchon sur un bout de terre propre et plat. Avec sa main droite, il prend du sable finement tamisé d' un petit tas qui se trouve derrière lui, à côté de ses basiw. Il étale ce sable sur le sol, sur une surface d' environ $75 \mathrm{~cm}$ sur $75 \mathrm{~cm}$. L' étalage du sable sur le sol se fait avec la main droite. Si le sable contient des morceaux, par exemple à cause du sang coagulé d' un sacrifice précédent, on peut utiliser les deux mains pour donner au sable sa structure normale. Une partie du sable reste sur le tas derrière l' expert. Il en aura besoin plus tard.

L' expert trace ensuite de la main droite un grand nimisa d' environ $50 \mathrm{~cm}$ sur $50 \mathrm{~cm}$ à l' intérieur duquel il place à droite un siké, à gauche un kumadibinè. Cela donne la figure 23 : 


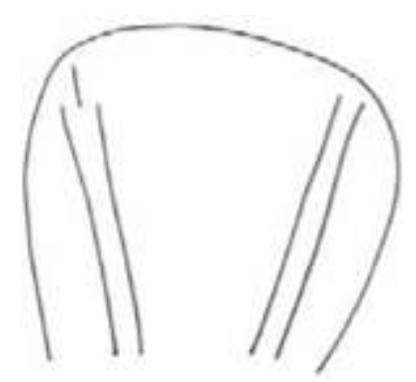

Fig. 23. - La PREMIÈre PHASE du SChÉma INAUGURAL garela(n). Cela donne comme résultat la figure 24 :

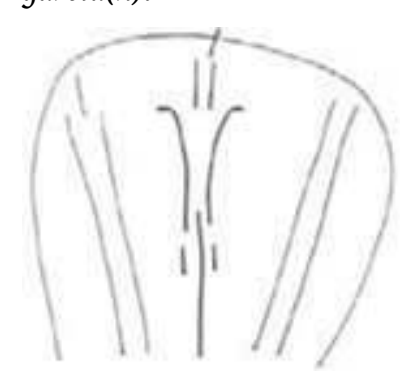

Fig. 24. - Le schéma INAUgURAL

$C^{\prime}$ est maintenant que commence la consultation proprement dite. Celui qui a fait le schéma prend dans sa main droite une poignée de sable du tas restant. Il tient cette main au-dessus du schéma et $l^{\prime}$ agite lentement comme un essuie-glace. Ensuite, il répand ainsi, petit à petit, le sable sur le schéma. Quand la main est vide, il prend encore une poignée de sable. Cette procédure s' appelle ka sènsèn (tamiser) ${ }^{26}$.

Pendant ce temps, la personne qui répand le sable continue à parler. Au cas où l' élève répand lui-même le sable, alors $c^{\prime}$ est le maître qui parle. Les mots sont prononcés rapidement. De cette façon, les phrases, qui sont du parler normal, résonnent comme des formules qu' on récite. La première phrase est bien connue : on y fait appel aux huit esprits et huit personnes (voir leçon 1). On parle régulièrement de Jitumu Bala (Bala originaire de Jitumu, une zone au sud de Bamako). Ce personnage est considéré, dans une grande partie du Mali, comme le fondateur légendaire de la géomancie ${ }^{27}$.

Avec ses mots, l' expert rend compte au sable (ka dantègè) : il dit qui il est, qui étaient ses maîtres, demande l' aide de ses maîtres, dit qu' il est honnête, qu' il ne cherche pas son intérêt personnel, qu' il n' a pas volé sa connaissance, ce qu' il a donné à son maître pour obtenir cette connaissance, quel genre de travail il a fait pour son maître et qu' il cherche les bénédictions du maître. Il nomme également les lieux où il est passé pour acquérir sa connaissance, la personne qui est maintenant en visite, quels voyages ce visiteur a faits. Très souvent, l' expert commence à se répéter après un certain temps, surtout quand il trouve nécessaire de se présenter des minutes durant au sable. Je veux dire qu' il fait une sorte $\mathrm{d}^{\prime}$ " impression management » et en même temps, une sorte de concentration; ainsi, le client est impressionné et au même moment cela donne au devin le temps de s' imprégner davantage de l' affaire. Il peut aussi ajouter des bénédictions pendant qu' il rend compte. Ceci est confirmé avec les amina (= «amen » [confirmation]) de l' assistance ${ }^{28}$. 
102 Avec ces mots, l' expert se présente non pas seulement au sable, mais aussi (indirectement) au client. Avec ses mots et ses bénédictions, il montre qu' il n' est pas un traficoteur, mais quelqu' un qui travaille dans une longue tradition et qui aidera le client avec dévouement.

103 Après cela, l' expert essuie avec sa main droite le schéma pour pouvoir recommencer « à zéro » avec la détermination des signes initiaux.

\section{Déterminer les quatre signes initiaux}

Ceux qui viennent consulter l' expert ont certaines plaintes ou désirs à déclarer ou pas. L' expert consulte le sable pour déterminer ce qui se passe dans la vie du visiteur. Il le fait en traçant un schéma de divination. Déterminer les quatre signes initiaux (ba naani = « les quatre mères ») est, pour un schéma de divination, une étape qui est composée d' une série d' opérations prédéterminées.

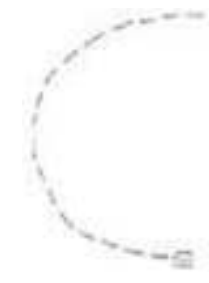

FIG. 25. - LE DÉBUt dU SCHÉMA POUR TROUVER UN SIGNE INITIAL

La même procédure se trouve quatre fois à la base de la détermination des quatre signes initiaux. Tout d' abord, l' élève pose trois doigts de sa main droite dans le sable et trace un trait à gauche. Ensuite il trace à l' aide de son index une courbe vers le haut en pointillés. Le nombre de traits $n^{\prime}$ est pas déterminé; il y en a toujours plus de treize et moins de vingt. $C^{\prime}$ est ainsi qu' on obtient, par exemple, un dessin comme dans la figure 25. On répète maintenant la même procédure pour obtenir deux courbes parallèles dont l' une est plus grande que l' autre (voir fig. 26).

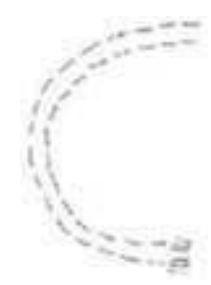

Fig. 26. - LE SCHÉMA POUR TROUVER UN SIGNE INITIAL, SUITE

106 Maintenant on trace, de chaque côté de la courbe, un arc de liaison partant des deuxièmes traits vers les quatrièmes traits, les sixièmes traits, et ainsi de suite, en continuant ainsi presque jusqu' à la fin de la courbe. Ainsi, on saura si le nombre de traits de chaque courbe est pair ou impair. Dans notre exemple, cela ressemble au suivant : 


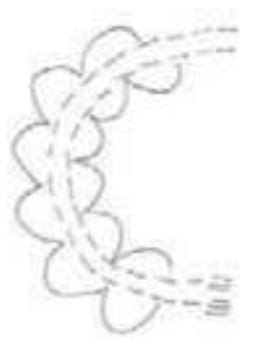

FIG. 27. - LeCtURE de LA COURBE : IMPAIR-PAIR

116 II

117 II

118 II

119 II

120

121 II

122 II

123 II

124 II

126

127

128

129 II

130 II

Dans notre exemple (fig. 27) la courbe extérieure a un nombre de traits pair et la courbe intérieure a un nombre de traits pair. À présent, l' expert applique une logique qui est déjà bien connue :

Impair = I

Pair = II

Il représente ce résultat respectivement dans les deuxième et quatrième positions d' un signe dans lequel les première et troisième positions sont paires. On obtient de cette façon le signe nimisa :

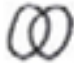

II ; connu d' avance, est par définition pair

II ; résultat de la courbe extérieure

II ; connu d' avance et est par définition pair

II ; résultat de la courbe intérieure

Si le résultat avait été impair-impair, alors cela aurait donné un jubidisè comme résultat II II

II

Un résultat pair-impair aurait donné un kumadisè II II II

Si le résultat avait été pair-pair, alors cela aurait donné un siké II II II II

En trouvant la figure 27, l' élève/l' expert aura découvert le premier signe, dans notre cas nimisa. Il « notera » ceci à droite de son schéma et effacera ensuite la figure. Ensuite, 
il reprendra encore trois fois la même procédure, et ainsi il obtiendra les quatre signes initiaux avec lesquels il peut faire un schéma de vingt-deux signes.

Il y a donc $4 \times 4 \times 4 \times 4=256$ schémas initiaux possibles parce qu' il y a seulement quatre différents signes initiaux possibles dans la première étape. Cela constitue une grande différence avec le système plus connu de divination avec seize cases où $16 \times 16 \times 16 \times$ $16=65536$ signes initiaux sont possibles.

Namagan connaît ces 256 schémas initiaux par cœur. En voyant les quatre signes initiaux, il montrait qu' il reconnaissait le schéma en disant les caractéristiques extérieures (voir plus bas). Il connaissait aussi les prochaines étapes de plusieurs schémas. Et pourtant, il accordait toujours beaucoup d' attention à l' élaboration de chaque schéma et sautait rarement une étape.

Un schéma peut être plus facile à retenir que l' autre. Cela peut être dû à des facteurs «esthétiques». Un exemple bien connu était le kumadisè-sikè-kumadisè-siké, quatre signes initiaux qui produisent huit signes jubidibinè. Ce qui donne une belle figure :

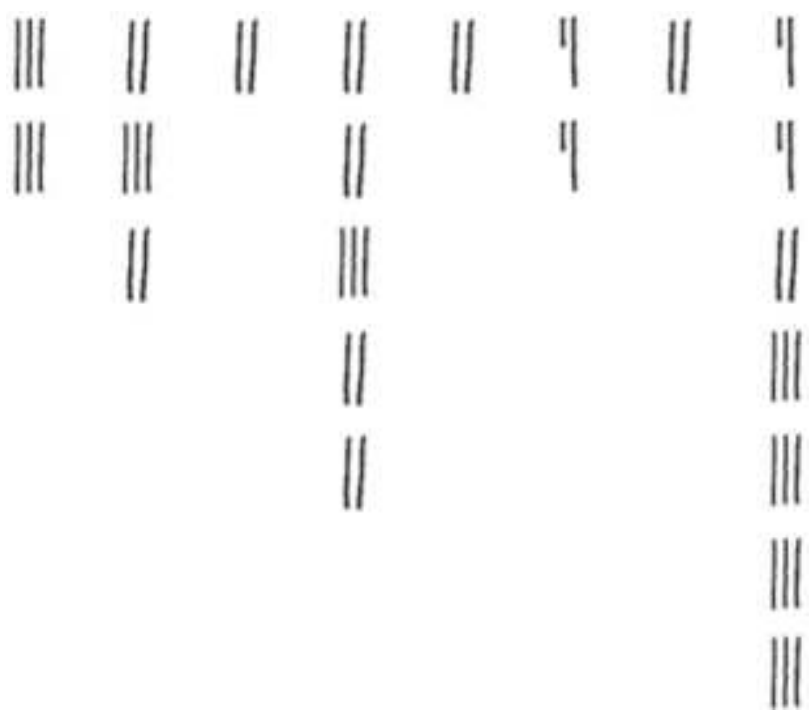

FIG. 28. - LE SCHÉMA QUI RÉSULTE DE KUMADISĖ-SIKĖ-KUMADISĖ-SIKÉ

$\mathrm{Au}$ début, je pensais que, pour déterminer les quatre signes initiaux, on pouvait faire appel à la manipulation, étant donné que le nombre de schémas, 256, est relativement peu élevé. Cependant, $j^{\prime}$ ai trois raisons de croire qu' il ne $s^{\prime}$ agit pas d' une manipulation:

Tout d' abord, ce n' est pas toujours l' expert qui fait les quatre signes initiaux; il fait souvent appel à un profane, généralement quelqu' un qui est là par hasard. Il est aussi possible que le client lui-même les ait effectués à la maison et vienne chez Namagan avec un papier comportant les quatre signes. De ce fait, on peut faire un schéma de divination aussitôt après la présentation au sable ${ }^{29}$.

La deuxième raison, pour laquelle je pense qu' il n' y a pas de manipulation, réside dans le caractère personnel de chaque consultation. $C^{\prime}$ est pourquoi, à $\mathrm{l}^{\prime}$ exception de quelques cas, les interprétations sont liées aux personnes; aussi bien Namagan que le vieux Bala me disaient que le choix d' un sacrifice ainsi que la position pour le sacrifice diffèrent selon l' individu. On peut donc trouver la variété dans l' interprétation en dehors du schéma, ou même en dépit du schéma. 
Une troisième raison est qu' il est contre-productif de manipuler les signes initiaux. On court ainsi le risque de cacher la vérité que le sable révèle. J' en donne comme exemple l' événement suivant.

Namagan lui-même a demandé, une fois, à un élève de produire un schéma avec siké et nimisa afin que se réalise "une bonne année ». L' élève a ensuite produit les signes initiaux kumadisè, kumadisè, jubidisè en siké. Cela donna la figure 29 comme résultat.

L' absence de nimisa dans ce schéma a poussé Namagan à conclure qu' il était question de «trahison (janfa) par un collègue ». Cet exemple montre que Namagan a intérêt à ne jamais manipuler les signes initiaux ; sinon il n' aurait jamais découvert la « trahison ».<smiles>C=C=C=CC(=C)C=C</smiles>

[Image non convertie]

Fig. 29. - Le schéma dans LEQUel NAMAgan SOUHAitait « NIMISA »

On détermine plus ou moins par hasard une combinaison de quatre signes initiaux. Même si c' est facile à réaliser, la manipulation ici est pratiquement absente parce que les signes initiaux sont réalisés par un non-initié, chaque schéma a une interprétation liée à une personne et toute manipulation est contre-productive.

Avec la recherche du sacrifice et sa signification, on a l' impression que les caractéristiques esthétiques sont très importantes. On est charmé par les belles formes dans le schéma ou par l' apparition fréquente d' un certain signe (voir aussi fig. 29) ${ }^{30}$. Cependant, la beauté est rarement le but principal de l' élaboration d' un schéma.

Selon moi, la beauté du schéma était tout de même un motif important lors d' une session, les 25-26 août 2002 - dont on me dit plus tard qu' il s' agissait de la " libération " ("labila») de Nanwali Kanté, un jeune homme de Farabako. Cette session commença avec, de droite à gauche, kumadisè, nimisa, siké, jubidibisè, et aboutit à l' étape 4 avec un motif formidable (voir fig. 33). Bien entendu, les experts savent cela à $\mathrm{l}^{\prime}$ avance! De plus, la quatrième étape est en même temps le "terminus ", parce qu' une éventuelle cinquième étape sera identique à la quatrième (voir fig. 33). Regardez maintenant les figures 30-33 et voyez comment naît, «tout d' un coup», un «joli» schéma.

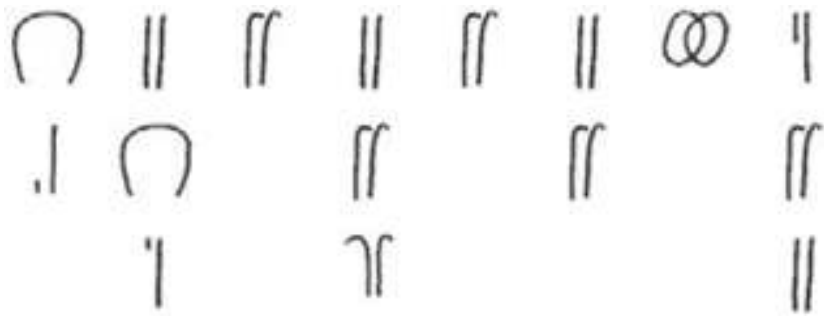

144 [Image non convertie]

FIG. 30. - LA « LibÉration » DE NANWALI, PREMIĖre ÉtAPE 


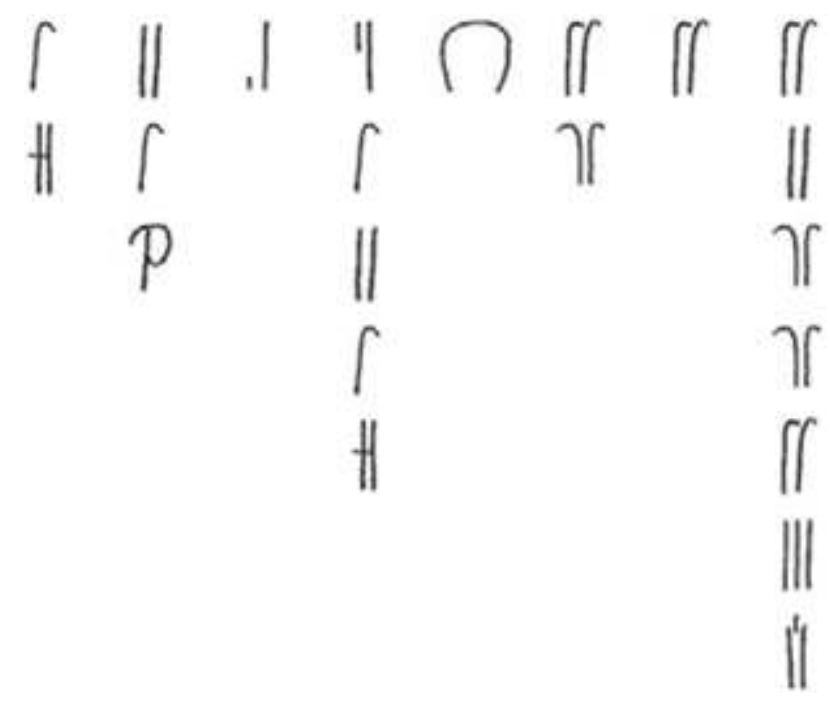

FIG. 31. - LA « LIBÉRATION » DE NANWALI, DEUXIÈME ÉTAPE

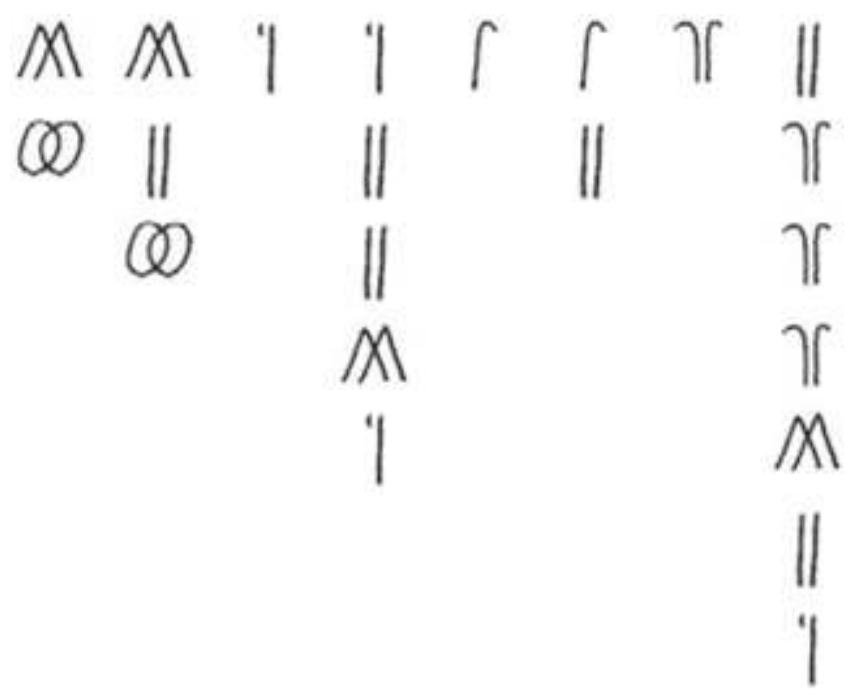

FIG. 32. - LA « LIBÉRATION » DE NANWALI, TROISIÈME ÉTAPE

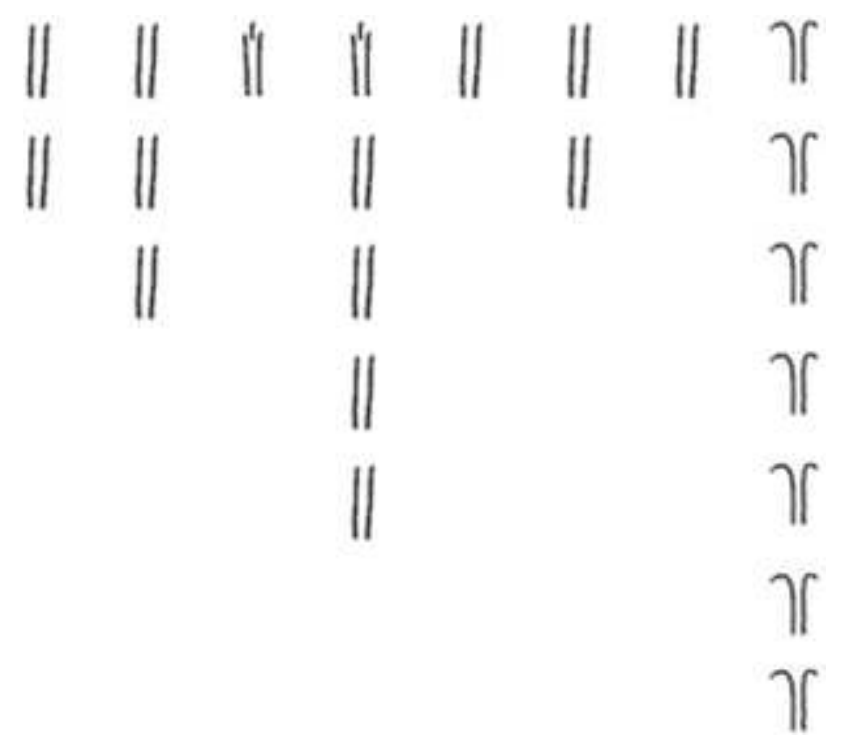

FIG. 33. - LA « LIBÉRATION » DE NANWALI, QUATRIÈME ÉTAPE 


\section{La modestie méthodique de l' expert et de son élève : un trajet d' apprentissage}

145 Les élèves avancés de Namagan ont remarqué que l' apprentissage des six leçons constitue la partie la plus importante de leur curriculum. Ils étaient $d^{\prime}$ avis que si quelqu' un pouvait faire sans faute les schémas dans le sable, il pouvait aussi librement consulter le sable. Ainsi, son maître le laissera «partir », le « libérera » ("ka labila»). Apparemment, ces élèves avancés trouvaient tellement que les autres aptitudes apprises allaient de soi, qu' ils considéraient la capacité de faire les schémas sans faute comme un critère pour " être libérés ».

Ces jeunes ne semblent pas s' apercevoir que le comportement, le parler, le mouvement, la connaissance des plantes et la connaissance des basiw sont aussi autant d' aptitudes nécessaires pour être un bon expert en géomancie; $c$ ' est un trajet d' apprentissage. Même après sa "libération", un élève ne doit jamais se présenter comme un expert ; il doit rester modeste. Namagan donnait lui-même le bon exemple. En présence d' un aîné ou d' un camarade du même âge qui connaissait aussi la géomancie, il se présentait toujours comme l' élève de ce dernier. Ce "maitre » ne protestait jamais contre cela, même s' il $n^{\prime}$ en pensait pas un mot. $C^{\prime}$ est surtout un signe de règles de politesse.

147 Un expert doit aussi être modeste par rapport à sa propre connaissance. Quand Namagan donnait la signification d' un schéma, il disait toujours qu' il l' avait appris de son maître Bakari Murukòrò Coulibaly, de Sananba Sèbèkòrò, un village des environs de Ségou. Ainsi, il place l' autorité de sa connaissance loin en dehors de la région dans laquelle il se trouve. Namagan estime la position de ses maîtres extrêmement importante. "Ton succès comme kinyèdala ("dessinateur de sable") est déterminé par le respect que tu accordes à tes maîtres ", me disait-il.

148 C' est seulement en août 2003, après plusieurs années de recherche que, assis derrière sa moto et lui posant des questions par rapport à ses maîtres (karamògòw), Namagan me donna une réponse qui me paraissait plus plausible : «J' ai beaucoup voyagé et partout, j' ai bien ouvert les yeux. Cependant, mes maîtres ont été, surtout feu mon père, Morifin (décédé vers 1995), Kinyèmadi (décédé au printemps 2003) et Bala (1931-10 janvier 2010). " Tous les autres hommes qui lui rendaient visite et qu' il me présentait comme étant ses " maîtres ", étaient en fait ses « élèves ». Cela correspondait à ce que je pensais, car $c^{\prime}$ était toujours Namagan qui «avait les choses en main» lors des sessions de divination avec ses « maîtres ».

149 La stratégie de modestie de Namagan est le comportement de base d' un expert en divination. En effet, ce dernier, dans les Monts Mandingues n' est pas quelqu' un qui a des connaissances encyclopédiques. Contrairement à la géomancie ifa, qui a été bien étudiée et où l' expert doit apprendre par cœur et appliquer un certain corpus bien donné, dans les Monts Mandingues l' interprétation diffère selon le client ${ }^{31}$. Lors du " début " officiel de mes «leçons ", en novembre 1999 auprès de Namagan Kanté, le vieux Bala Kanté disait: «Une seule personne ne peut pas tout comprendre » (« Mògò kilin $t^{\prime}$ o ban nyè dò la»). Un expert est donc, en partie, dépendant $\mathrm{d}^{\prime}$ une bonne présentation et $d$ ' une oreille attentive (voir note 6). 
Par conséquent, l' expert opère avec modestie et circonspection. Alors que Namagan, en présence de «jeunes » élèves et visiteurs, donnait sans hésiter des prescriptions et des interprétations, il s' en abstenait en présence d' autres experts. Après avoir dessiné (da) le vingt-deuxième signe, les experts regardaient toujours pendant quelques secondes le schéma avant que l' un d' entre eux prenne la parole. Si personne ne le faisait, alors $c^{\prime}$ est Namagan qui donnait la parole à quelqu' un en posant une question. D' emblée, il était toujours $d^{\prime}$ accord avec la réponse, même s' il y ajoutait souvent quelque commentaire. Il est donc clair qu' il y a, ici, de la place pour un peu de créativité.

À part la modestie, il est aussi nécessaire d' avoir de la circonspection. On peut faire cela par exemple en affirmant qu' il est vrai qu' on connaît quelque chose, mais qu' on ne peut pas le dire parce que c' est un secret personnel (dalilu). Moussa Kanté, le fils aîné du fameux (Farabako-)Jigin (décédé en 2001), faisait régulièrement cela. Un peu plus tard, peut-être sous l' influence de l' alcool, ou parce qu' il avait constaté que personne $\mathrm{d}^{\prime}$ autre $\mathrm{n}^{\prime}$ avait rien dit, Moussa donnait toujours l' interprétation "secrète ».

152 Étant donné que la géomancie dans les Monts Mandingues "ne connaît que » 256 schémas initiaux (voir leçons 4-6), les experts ont déjà vu souvent tous les schémas initiaux possibles ainsi que les étapes suivantes. Puisqu' un certain schéma peut être la spécialité d' un autre expert, il est dangereux d' interpréter à l' improviste sans réfléchir. J' ai testé cette hypothèse en présentant à Namagan quatre signes pris au hasard. Il compléta immédiatement le schéma qui s' y rapportait. Mais après un moment de silence, il me dit qu' il ne pouvait rien faire avec ce schéma, parce que son «maitre ne lui a pas appris cela ». Il n' a donc pas exclu l' existence du schéma et/ou son manque de signification ${ }^{32}$. Il ajouta cependant, après un moment de réflexion, et comme à contrecœur, qu' il fallait "sacrifier du haricot à l' unique katé du schéma. Après avoir cuit ce haricot, on doit le manger et aller au lit sans se laver les mains. Cela porte de la chance ". J' ai souvent entendu Namagan donner une telle " recette » à des visiteurs. Il dit aussi, quelque temps plus tard, qu' à l' unique nyagaransè du schéma, il fallait sacrifier un tissu (fanni) et une noix de cola (wò̀ò) - un principe bien connu pour éviter qu' une certaine femme ne marie un homme donné.

Avec ce test, Namagan ne sortait pas du cadre que je lui connaissais; il renvoyait à son maitre "Bakari » et, pendant qu' un chercheur avait un doute méthodique comme seconde nature, Namagan, lui, avait une modestie méthodique. Cependant, cela $n^{\prime}$ empêchait pas que ses commentaires et interprétations soient également très directs et concrets (tout comme l' homme de science est souvent très convaincu de ses points de vue). $C^{\prime}$ est de cette grande variation dans le « don des impressions » que l' expert tire en partie son statut, pas seulement dans le domaine de la divination mais aussi dans d' autres formes d' expertise ${ }^{33}$.

154 Dans la plupart des cas, l' expert connaît effectivement à peine son client. La raison en est donnée par Colleyn (2005): il est en effet dangereux d' impliquer dans tes problèmes quelqu' un qui connaît ta famille. $C^{\prime}$ est pourquoi les gens cherchent leur salut chez un expert d' un autre groupe ethnique. Quant à l' expert lui-même, il a reçu sa connaissance en dehors de son propre groupe. L' expert compose sur place un complexe impressionnant de « remèdes » et de règles de conduite. La connaissance est crédible parce que, selon l' approche de Colleyn (ibid.), aussi bien l' expert que le client 
associent de la même façon les unes aux autres les règles sociales et les lois cosmologiques $^{34}$.

Il manque à mon approche les préparatifs personnels d' un devin, parce qu' ils doivent rester cachés ou jouer l' isolement. Je sais que Namagan consulte souvent tout seul le sable. «Sans baara [travail, pratique, exercice], on perd la main », m' a-t-il dit une fois, en me montrant la face poussiéreuse de sa main droite.

À travers le comportement du maître, l' élève apprend à imiter non seulement en mots, mais aussi en gestes (Marchand 2001, 2009, ch. 5). L' attention avec laquelle l' expert traite ses outils apparait non seulement dans ce qu' il dit, mais aussi dans tous ses mouvements. On dirait que l' expert «sent» comment et quand il doit faire quelque chose. Par exemple, lors de sa "session de libération ", Nanwali (voir ci-dessus) devait sacrifier sept noix de cola blanches et rouges aux sept signes nyagaranbinè qu' il reçut finalement dans la quatrième étape (voir fig. 33). Il les posa, comme si cela allait de soi, dans les sept fois deux "aisselles » des sept signes nyagaranbinè. Après le sacrifice des feuilles aux noix de cola, il les tira à lui des deux mains dans un mouvement ininterrompu et lent. Nanwali $\mathrm{m}$ ' a impressionné par l' intensité et le calme avec lesquels il opérait.

Au fil des années, j' ai été impressionné par le soin et l' attention minutieux, et souvent exagérés, avec lesquels un devin faisait son travail. Pour faire un objet de pouvoir (basi), il faut compter plusieurs heures. Par exemple, il faut $d^{\prime}$ abord nettoyer une corne de bélier, ensuite limer ses côtés rugueux et après mettre dans la corne un objet ou un morceau de papier écrit. Pour finir, il faut y crachoter (tu) des bénédictions, fermer la corne et l' entourer d' un fil de coton et, pour parfaire le tout, faire dessus un sacrifice de telle sorte que le basi reçoive une couche de sang coagulé. Chacune de ces opérations est exécutée par l' expert avec beaucoup de précaution et de précision. Par exemple, en sacrifiant une poule, il doit éviter de couper une seule plume.

L' expert donne aussi des instructions précises au client pour que celui-ci se comporte correctement pendant le sacrifice. Le client doit, le plus souvent, poser sa main droite sur la poule ou les feuilles qui sont sacrifiées, pendant que l' expert parle. Souvent, le client doit se tenir debout dans le schéma de sable, de sorte qu' il est littéralement incorporé dans le sacrifice et le sable. Cela donne, bien entendu, une grande impression d' intensité.

159 Je considère l' attention pour l' outil du sacrifice comme une forme de soin que le client mérite selon le "code de profession » de l' expert. Ce code n' est pas spécifique aux experts en divination des Monts Mandingues; on le retrouve par exemple chez les experts médicaux du début des temps modernes. Porter (2002:37-38) écrit ce qui suit sur les docteurs « hippocratiques » de cette période, quand la connaissance biomédicale était encore limitée et que beaucoup de médecins devaient surtout se contenter des soins qu' ils pouvaient offrir :

«[...] Le médecin des temps anciens devait faire un choix entre les options conservatives hippocratiques (attendre et observer, le repos, les remontants, le soin, les mots de consolation, le calme et l' espoir) et les possibilités "héroïques", y compris les purges violentes, les saignées drastiques (la préférence de Galenus) ou un autre remède de bonne femme. Souvent, la décision était prise pour lui : des patients grincheux avaient de fortes options sur le meilleur traitement pour "leurs" maladies, et comme c' étaient eux qui payaient, il fallait faire ce qu' ils voulaient. » 
La description que fait Porter des médecins de la société européenne du début des temps modernes est comparable à la conception de Namagan (voir «par qui, pour qui ») selon laquelle il y a trois sortes de relations, et le client, à travers son propre diagnostic des causes de son mal, détermine la durée de la relation.

$L^{\prime}$ accomplissement $d^{\prime}$ un sacrifice $s^{\prime}$ accompagne de certains mouvements et attitudes ${ }^{35}$. Lors de $\mathrm{l}^{\prime}$ interprétation et de $\mathrm{l}^{\prime}$ élaboration du schéma de divination, $\mathrm{l}^{\prime}$ expert est assis sur le sol, les deux jambes droites devant et le corps légèrement tourné à droite vers le sable, alors que durant le sacrifice il est plutôt accroupi, avec ses pieds à plat sur le sol - une attitude que l' on apprend depuis l' enfance. L' expert crache (tu) quand il prononce une formule magique (kirisi/kilisi). Il crache le plus souvent sur l' objet qui est sacrifié ou sur le basi qu' il fabrique, mais il peut tout aussi bien cracher sur le couteau avec lequel on égorge l' animal sacrifié. Le crachat est composé le plus souvent de salive, mais souvent aussi d' alcool ou de noix de cola mâchée.

Le fait de cracher les mots souligne la valeur et la force du mot prononcé. La preuve de la force des mots m' a été donnée quand Namagan $\mathrm{m}$ ' a permis d' enregistrer ses kirisiw (pluriel de kirisi) et de les copier (phonétiquement) dans mon cahier de notes, mais il ne $\mathrm{m}$ ' a pas donné la permission de les publier. Et pourtant, il ne se faisait jamais de souci par rapport à d' autres informations qu' il me donnait ${ }^{36}$.

La force de la parole du devin se manifeste aussi dans les longues bénédictions incantations et présentation au sable $d^{\prime}$ une session. Par rapport à cela, j' avais $\mathrm{l}^{\prime}$ impression que la durée des paroles était déterminée, d' un côté par l' importance de l' affaire et, de l' autre, par l' intensité de la relation entre l' expert et son client.

Beaucoup d' opérations ont produit sur moi une impression violente mais, à l' instar de Porter, dois-je les appeler "héroïques»? Le plus souvent, les devins projettent les poulets sacrifiés un peu plus loin, par exemple au milieu de la cour où les bêtes se débattent contre la mort. Avant cela, on asperge le sable et le basi du sang de la poule. Par exemple, en août 2003, Namagan avait secoué longuement la poule qu' il venait d' égorger pour la sacrifier à Danbakoriya (stop à la trahison), un basi avec une corne de bélier comme support que Namagan avait faite pour moi afin de me protéger contre la rivalité des collègues. "Le basi demande du sang», $\mathrm{m}$ ' expliqua-t-il. Le fait aussi de déchirer quelques centimètres du bec de la poule me donna des frissons : je connais l' opinion des gens sur la force de la parole et cette "réduction au silence " $\mathrm{m}^{\prime}$ impressionna beaucoup. Je fus surtout effrayé de la chèvre qui, à l' improviste, était jetée de la fenêtre tout juste au-dessus de ma tête $-\mathrm{j}$ ' étais assis dehors, sous la fenêtre - lors d' une session dans la chambre de (Farabako-) Jigin durant laquelle les experts de Farabako devaient, ensemble, retrouver l' identité de certains voleurs de bétail.

Ce ne sont pas seulement les actions, mais aussi les paroles qui peuvent être «héroïques ». Par exemple, en août 2003, avant une promenade en "brousse", Namagan avait, dans sa propre case, sacrifié sept morceaux de bois (avec un bout pointu) sur chacun des sept signes maromaro dans un schéma de divination. Ce sacrifice avait pour but de permettre le paiement du reliquat de salaire d' un client venant de Bamako. Namagan enfonça les sept bâtonnets, à quelques centimètres de distance l' un de l' autre, dans un arbre un peu en dehors de la ville. Cela eut comme effet de fendiller l' écorce entre les sept bâtonnets. En même temps qu' il enfonçait les bâtonnets, il se mit à proférer des insultes grossières et même des menaces : « $S$ ' il est vrai que ton chef mange avec la bouche et se sert de son anus pour chier, il te payera. Si au contraire, il 
mange avec son anus et fait caca avec la bouche, il ne te payera point. " Namagan m' expliqua : "Maromaro (bè) ko minè a tigi la » ("Maromaro va l' obliger »; littéralement "Maromaro enlèvera l' affaire des mains de son propriétaire »). $\mathrm{Il} \mathrm{m}$ ' expliqua que le chef aurait seulement la conscience tranquille quand les fissures entre les bâtonnets se seront recomposées. Ce qui veut dire que le sacrifice $n^{\prime}$ est pas absolu, mais a un effet temporaire.

D' après la terminologie de Porter, $c^{\prime}$ est la combinaison de deux choses, « prendre soin de » et l' aspect "héroïque», qui font de la session de divination un événement personnel, intensif et impressionnant pour le client. Ce dernier est tiraillé (ou même affolé) entre tranquillité et connaissance d' un côté, opposées à violence et action de l' autre. Par exemple, après avoir minutieusement déterminé la couleur de la poule (ou du coq), lui avoir tranché le cou et l' avoir projetée par terre, on va ensuite discuter longuement sur la façon dont les pattes de la poule sont disposées.

\section{Quelques remarques sur le « savoir-faire »}

La région de Sobara, au milieu des Monts Mandingues, a une position marginale dans l' économie de la zone au sud de Bamako, la capitale du Mali. Le système de divination que $l^{\prime}$ on utilise là-bas a une étendue géographique limitée. Son origine $\mathrm{n}^{\prime}$ est pas connue, mais il s' agit certainement $d^{\prime}$ une variation du fameux système des seize cases connu dans une grande partie du monde. Ce qui rend unique cette forme de divination est le système d' enseignement arithmétique de six leçons.

La divination est une activité qu' on pourrait, peut-être, totalement connaître ; mais pour cela il faudrait la pratiquer activement (Graw 2005). Je n' en suis pas arrivé là et je n' y arriverai jamais. Cependant, j' ai de la chance car un certain nombre d' aspects de la divination sont élaborés en un système $d$ ' enseignement formalisé. Cela est $d$ ' autant plus inattendu dans un contexte où, jusqu' à récemment, les écoles occidentales et coraniques étaient absentes. Heureusement qu' il $\mathrm{n}^{\prime} \mathrm{y}$ a pas de problèmes pour publier ce système $\mathrm{d}^{\prime}$ enseignement formalisé. $\mathrm{J}$ ' assiste aux sessions des experts à Farabako pour apprendre leur « savoir-faire ».

169 " $N^{\prime}$ i y' a faamu, i y' a faamu dò̀ni dòòni " ( Si tu l' as compris, tu ne l' as compris qu' un tout petit peu »). Ces mots viennent $d^{\prime}$ un chauffeur de taxi, Madou Doumbia, qui voulait $m$ ' expliquer à quel point sa propre culture (bamanaya) était complexe. Je pense qu' une telle modestie est de mise pour tout homme qui étudie la géomancie.

\section{BIBLIOGRAPHIE}

AsCHER, M. 1997 " Malagasy Sikidy: A Case in Ethnomathematics », Historia Mathematica, 24 :

376-395.

Ashforth, A. 2000 Madumo, a Man Bewitched, Chicago-London, Chicago University Press. 
Austen, R. A. 2008 " The Problem of the Mande Creation Myth ", in S. BELCHER, J. JANSEN \& M. N' DAOu (eds.), Mande Mansa : Essays in Honor of David C. Conrad, Zürich-Münster, Lit Verlag : 31-39.

BAILLEUL, C. 1996 Dictionnaire Bambara-Français, Bamako, Donniya.

BERTAUX, C. 1983 " La technique des prescriptions sacrificielles dans la géomancie bambara (région de Ségou, Mali) », Systèmes de pensée en Afrique noire, 6 : 117-130.

BRENNER, L. 2000 " Muslim Divination and the History of Religion of Sub-Sahara Africa ", in J. PEMBERTON (ed.), Insight and Artistry in African Divination, Washington-London, Smithsonian : 45-59.

Colleyn, J.-P. 2005 " La géomancie dans le contexte bamana. Signes et objets forts ", Mande Studies, $7: 9-20$.

Derive, J. \& DUMESTRE, G. 1999 Des hommes et des bêtes. Chants de chasseurs mandingues, Paris, Association des Classiques africains.

Dieterlen, G. 1957 ” The Mande Creation Myth », Africa, 27 : 124-138.

EGLASH, R. 1997 " Bamana Sand Divination ", American Anthropologist, 99 (1) : 112-122. 1999 African Fractals. Modern Computing and Indigenous Design, New Brunswick, NJ, Rutgers ; London, New Press University.

GESCHIERE, P. L. 2000 " Sorcellerie et modernité : retour sur une étrange complicité », Politique africaine, $79: 17-32$.

GRAW, K. 2005 " The Logic of Shells : Knowledge and Lifeworld-Poiesis in Senegambian Cowrie Divination », Mande Studies, 7 :21-48.

JANSEN, J. 2002 Les secrets du Manding. Les récits du sanctuaire Kamabolon de Kangaba (Mali), Leiden, Research School CNWS.

JANSEN, J. \& DIARRA, M. 2006 Entretiens avec Bala Kanté. Une chronique du Manding du XXe siècle, Leiden-Boston, Brill.

JANSEN, J. \& KANTÉ, N. 2010 La géomancie des Monts Mandingues. L' art de lire l' avenir dans le sable, Bamako, Yeelen.

JAULIN, R. 1966 La géomancie. Analyse formelle, Paris-La Haye, Mouton.

KASSIBO, B. 1992 " La géomancie ouest-africaine. Formes endogènes et emprunts extérieurs ", Cahiers d' Études africaines, XXXII (4), $128: 541-596$.

MARCHAND, T. H. J. 2001 Minaret Building and Apprenticeship in Yemen, Richmond (Surrey), Curzon. 2009 The Masons of Djenne, Bloomington-Indianapolis, Indiana University Press.

Mcnaughton, P. R. 1988 The Mande Blacksmiths : Knowledge, Power, and Art in West Africa, Bloomington-Indianapolis, Indiana University Press.

PEEK, P. M. 1991 African Divination Systems. Ways of Knowing, Bloomington-Indianapolis, Indiana University Press.

PORTER, R. 2002 Blood and Guts. A Short History of Medicine, London, Penguin. 


\section{NOTES}

1. Un grand merci à tous les experts en géomancie à Farabako et à leurs familles. Farabako est un petit village d' environ quatre cents habitants, où j' ai fait mes recherches de terrain durant la période juillet 1999-novembre 1999, mars 2000, août 2002, août 2003, novembre 2004, janvier 2006 et janvier 2007. Farabako est situé dans la zone de Sobara (arrondissement de Siby), au milieu des Monts Mandingues. Je remercie Moussa Fofana pour la traduction du néerlandais et la correction du français. Je remercie Brahima Camara, Jean-Paul Colleyn, Moussa Fofana, Paulus Gerdes, Trevor Marchand, Philip Peek, Boubacar Tamboura et Walter van Beek pour leurs commentaires.

2. KASSIBO (1992) parle d' un système avec vingt-deux cases, mais tel n' est certainement pas le système des Monts Mandingues. Par rapport au chiffre 22, il est remarquable qu' il est à la base du "The Mande Creation Myth ", un article fameux de DIETERLEN (1957) dont le statut scientifique est incertain (AUSTEN 2008 ; JANSEN 2002). En dépit de l' origine ancienne du système des seize cases, VAN BINSBERGEN (communication personnelle, juin 2005 ; voir aussi son œuvre sur <www.shikanda.net>) affirme que les écrits arabes (islamiques) du Moyen-Âge sur ce système ont eu tellement d' influence dans une grande partie du monde $\mathrm{qu}^{\prime}$ on pourrait parler de globalisation. Il marque ses influences dans beaucoup $\mathrm{d}^{\prime}$ autres systèmes et $\mathrm{c}^{\prime}$ est aussi le cas de la géomancie dans les Monts Mandingues (voir leçon 2). Van Binsbergen appellerait ce système "un système fragmenté local de géomancie », et les gens utilisant ce système de géomancie $\mathrm{n}$ ' auraient (plus) aucune conscience des racines transrégionales et islamiques de leur système (ibid.).

3. BERTAUX (1983) apporte une importante contribution ethnographique. KASSIBO (1992) a écrit un article qui donne une vue d' ensemble de la littérature sur ce système, mais cet article n' aborde pas la position sociale et culturelle des experts en géomancie. Des chercheurs contemporains tels que BRENNER (2000) et VAN BINSBERGEN (<www.shikanda.net $>$ ), abordent bien ces aspects.

4. Les experts en géomancie des Monts Mandingues réfutent donc - comme l' avait prédit van Binsbergen - les « racines transrégionales » de leur système.

5. Les termes tinyè (vérité) et kinyè (sable) sont, aussi bien en maninka qu' en bambara, prononcés comme " cèn " (BERTAUX 1983 : 117). Cela donne aussi une certaine valeur ajoutée à la géomancie.

6. En outre, j' ai beaucoup appris quand mon maître Namagan Kanté $m$ ' a appelé «sur la peau [de chèvre]» (golo kan) pour corriger et commenter mes opérations. Pour une description de ces expériences - dans laquelle je mets un accent sur les manifestations (" appearances » dans le vocabulaire de Goffman) - voir la deuxième partie de JANSEN et KANTÉ (2010).

7. Je suis toujours resté un étranger. Une bonne illustration en est la plainte contre moi qu' a déposée un collègue élève. Ce dernier a demandé à Namagan : « Pourquoi ce Blanc a-t-il la permission de faire des fautes et de poser des questions? Quand c' est nous qui le faisons, vous nous chassez ou nous tapez avec un fouet. " Namagan (qui est né en 1964, donc de ma génération) donna la réponse suivante : "Je le considère comme un vieux [qu' on ne doit ni contredire ni battre], avec le respect qu' un vieux mérite. »

8. Namagan Kanté, 14 août 2003. 
9. La matérialisation de ce grand réseau de Namagan est sa moto. Namagan était la seule personne à Farabako possédant une moto avec un moteur puissant qui, même pendant la saison des pluies, pouvait le conduire en dehors de la région.

10. Namagan explique sa préférence pour les vaches en faisant savoir qu' enfant il avait les yeux enflés et rouges chaque fois qu' il se rendait dans un champ de mil. À cause de cette " allergie » - il porte souvent des lunettes de soleil - Namagan était obligé de se spécialiser dans une activité non traditionnelle. Cette spécialisation coïncide historiquement avec une augmentation du nombre de vaches dans la région (JANSEN \& DIARRA 2006).

11. Cette leçon sur la patience est sans doute liée à la vision critique que Namagan a de l' utilisation que font les Blancs (tubabuw) du «temps ».

12. Lors de ma recherche sur le terrain, $\mathrm{j}^{\prime}$ ai eu l' impression que l' aspect binaire (I ou II) n' était jamais un point d' intérêt pour Namagan et ses collègues; ils cherchaient la signification au niveau des signes et leurs relations mutuelles dans le schéma de géomancie.

13. BERTAUX (1983 : 118) parle aussi des huit esprits et huit personnes dans la géomancie bamana, mais selon lui, contrairement à ce qui est décrit ici, les huit personnes sont toujours nommées les premières, et après viennent les huit esprits.

14. Pour être exhaustif : il y a une petite différence partielle de nature technique. Si tèrèmèsè se trouve sous un signe féminin - tèrèdibinè, nyagaransè, nyagaranbinè et katé-, le tèrèmèsè est tourné à 180 degrés avec comme résultat :. Namagan (11 août 2003) ne pouvait pas $\mathrm{m}^{\prime}$ expliquer pourquoi ces quatre signes étaient «féminins ». À partir de mes propres observations, $\mathrm{j}^{\prime}$ en ai déduit qu' également sous un tèrèmèsè renversé, un tèrèmèsè est tourné à 180 degrés.

15. On écrit katé différemment sur papier que dans le sable. Dans le sable, on l' écrit comme un siké, mais avec une plus petite distance entre les deux traits verticaux dans siké, la distance entre les traits est d' environ $3 \mathrm{~cm}$. J' avais toujours des difficultés à faire la différence entre siké et katé dans le sable, et pourtant Namagan $\mathrm{m}$ ' interdisait d' ajouter un petit trait horizontal en écrivant un katé dans le sable. La conséquence en était que lors de l' analyse, je me trompais souvent entre siké et katé.

16. Cela correspond à la remarque de BERTAUX (1983:118), sur le système de géomancie semblable chez les Bamana : "Ces êtres géomantiques, à l' état de calligramme sous le tableau divinatoire, peuvent se percevoir en brousse sous la forme $d$ ' êtres fantastiques : nains, géants, personnes humaines vivant en brousse. »

17. En raison de l' importance du terme dibi dans la cosmologie, je me suis souvent demandé si j' avais bien compris jubidisè et jubidinè. $\mathrm{N}^{\prime}$ était-ce pas plutôt judibisè et judibinè ? Quand j' ai demandé, on $\mathrm{m}$ ' a répondu que ce $\mathrm{n}^{\prime}$ était pas le cas.

18. Communication personnelle, août 2005.

19. Mes maîtres désapprouvaient toujours quand j' utilisais le terme calcul (jate). Eux utilisaient toujours des termes comme kalan (étudier, lire et da [former, (ex)poser]). Dans ma description, je parlerai du regroupement des signes.

20. À la position 19, il ne peut y avoir que huit des seize signes parce que le dernier «bit » du code binaire est toujours II ; le dernier « bit » est en effet toujours pair, étant donné qu' il résulte du regroupement de deux fois le même bit (le dernier de la position douze). J' ai souvent fait une telle observation à Namagan, mais il réagissait avec un 
haussement d' épaule à cette remarque qui se voulait "intelligente »; une telle chose est visiblement sans importance pour lui.

21. La même problématique est aussi décrite par ASCHER (1997) qui utilise une description mathématique que préfère Paulus Gerdes (communication personnelle, automne 2005).

22. Il est peut-être superflu de mentionner que Namagan lui-même a reconnu immédiatement l' explication de mes règles "arithmétiques ", mais en même temps il $\mathrm{m}$ ' a donné l' impression que la chose allait tellement de soi qu' elle ne méritait pas qu' on y accordât de l' importance.

23. Pour une description de la neutralité arithmétique qui est en vigueur en choisissant siké, nimisa, kumadisè et jubidisè comme signes initiaux, voir JANSEN et KANTÉ (2010, Appendice II).

24. En cela, j' estime que le système des Monts Mandingues est plus subtil que celui, plus connu, de seize cases parce que dans ce cas, on prend les quatre derniers signes comme des signes initiaux pour un prochain schéma (BERTAUX 1983 : 119).

25. La deuxième étape - la plus simple - est sans doute celle de la leçon 3. Ce schéma, qui est fréquemment utilisé, est composé de 22 signes siké, un schéma qui est considéré comme mauvais (jugu) (voir la première étape de la deuxième partie, sur les «33 hommes ", dans JANSEN et KANTÉ [2010]).

26. On prenait le plus souvent la figure 24 comme point de départ. J' ai été témoin une seule fois quand on a pris la figure 23. La différence entre ces stratégies m' échappe.

27. Pour une légende sur Jitumu Bala, voir BERTAUX (1983 : 129).

28. Il est intéressant de mentionner que Boubacar Tamboura faisait souvent le commentaire qu' une telle présentation était de l' esbroufe et que le plus important était les feuilles/potions que le client devait recevoir. Je trouve cette critique inattendue de la part d' un Malien dont j' attends plutôt qu' il ne sous-estime pas la force de la parole.

29. Souvent, quelqu' un peut justement manipuler les signes de façon explicite. Ainsi j' ai pu voir, en août 2003, comment un beau-frère de Namagan (pas un "expert ", mais plutôt un profane) recommença à faire quatre signes initiaux parce qu' il ne voulait pas avoir de nimisa parmi les quatre signes initiaux. Mais cela ne veut pas dire qu' il ne doit pas y en avoir plus loin dans le schéma.

30. EGLASH $(1997,1999)$ se laisse inspirer par les «fractales» et, de ce fait, met la géomancie (avec seize cases) en rapport avec des modèles graphiques qui se répètent sur une échelle de plus en plus petite (16-8-4-2-1). Cet aspect esthétique de la " fractale»- sur lequel selon moi Eglash met trop l' accent, ce qui fait qu' il sort la géomancie de son contexte de production - est bien entendu absent du système des vingt-deux positions.

31. Selon les ouvrages sur la géomancie bamana, j' ai l' impression que les sacrifices et les schémas sont formellement prédéterminés (BERTAUX 1983). Cependant, je suppose aussi que, dans la pratique, les experts bamana font souvent une interprétation au cas par cas.

32. Mon schéma imaginaire était composé de trois tèrèmèsè et $\mathrm{d}^{\prime}$ un quatrième signe que j' ai oublié. Étant donné qu' un expert fait au maximum sept étapes, il était 
invraisemblable que ce schéma appartienne aux $1792(256 \times 7)$ schémas qu' il ne verra jamais dans sa vie. Namagan $n$ ' apprécie pas de tels arguments arithmétiques.

33. Selon le dictionnaire néerlandais Wolters, un (expert) devin est quelqu' un qui rend un jugement sur des affaires controversées. $C^{\prime}$ est pourquoi j' appelle Namagan un (expert) devin et non pas un spécialiste ; un spécialiste est quelqu' un que l' on appelle seulement pour ses connaissances ou ses capacités.

34. J' aurais pu tester mon approche avec une étude systématique de l' interprétation du schéma, mais pour cela il me manque le temps et les capacités linguistiques. Mon étude est donc " provisoire ", mais je me sens réconforté par l' analyse de Colleyn. GRAW (2005) donne la primauté à l' interaction entre le devin et son client et, ainsi, rend absolu le caractère relatif et relationnel de la connaissance de l' expert. Pour moi, il n' est cependant pas clair que ce que l' expert veut dire a une certaine relation avec ce que le client désire et vice-versa.

35. Les mouvements sont, je suppose, déterminés sur une base régionale ou locale. Boubacar Tamboura me fit remarquer qu' à Farabako, on finit généralement la présentation au sable avec un claquement de doigts alors qu' ailleurs il est courant de le faire avec un balancement de la main vers le haut.

36. C' était là la seule interdiction définitive à laquelle j' ai eu affaire à Farabako.

\section{RÉSUMÉS}

RÉSUMÉ

Cet article introduit un cheminement d' apprentissage formalisé de géomancie. L' apprentissage démontre, contre ce que les théories proposent, qu' un enseignement arithmétique standardisé peut exister dans une société qui n' a développé aucun système mathématique, qui est à peine alphabétisée, et dans laquelle l' écriture est surtout une technologie dont on a besoin pour la reproduction des textes religieux et non pas pour l' organisation de la société. En même temps, l' article donne une (première !) description formelle du système des Monts Mandingues, qui est un système avec vingt-deux «cases». La numérologie de ce système peut ouvrir de nouvelles perspectives sur des textes mandingues « classiques » des mythes de création.

\section{ABSTRACT}

Arithmetic Education as Apprenticeships. Geomancy in the Mande Mountains.

This article describes a trajectory of formalized apprenticeship in divination. This apprenticeship demonstrates, in contrast to what theories predict, that formalized arithmetic education can exist in a society that has not developed a system of mathematics, that has a low level of literacy, and in which writing is mostly a technology used for the reproduction of religious texts (and not for the organization of the society). At the same time, this article describes the hitherto never noticed divination system of the Mande Mountains, which is a system with twenty-two "houses". The numerology of this system may offer new perspectives on "classical" Mande texts of creation myths. 
INDEX

Mots-clés : Mali, Sobara, Mandé, Monts Mandingues, Griaule, Dieterlen, arithmétique, divination, éducation, géomancie, leçons, mathématique

\section{AUTEUR}

\section{JAN JANSEN}

Institut d' Anthropologie culturelle et de sociologie du développement, Université de Leiden, Pays-Bas. 